\title{
Zur Messung der Nutzung regionaler Potenziale beim Ausbau erneuerbarer Energien. Eine empirische Analyse deutscher Landkreise
}

\section{How to measure the usage of regional potentials of renewable energies. An empirical analysis of German counties}

https://doi.org/10.2478/rara-2019-0043

Eingegangen: 28. September 2018; Angenommen: 24. Juni 2019

Kurzfassung: Der Beitrag beantwortet zwei bislang kaum behandelte Fragen: Wie gut schöpfen deutsche Regionen inr Potenzial zur Erzeugung regenerativer Energien aus und welche Faktoren beeinflussen diese Ausbaueffizienz? Mit dem neuartigen quantitativ-empirischen Konzept der Ausbaueffizienz wird das Ausmaß ermittelt, mit dem Regionen ihre natürlichen und sozioökonomischen Potenziale zur Erzeugung von Energie aus erneuerbaren Quellen zu einem bestimmten Zeitpunkt ausgeschöpft haben. Dieser Ansatz erlaubt unter Berücksichtigung der Energieträger Wind-, Solar- und Biogasenergie einen relativen Vergleich von Regionen, ihre Ausbaueffizienz über die Zeit sowie die Identifikation von Best-practice-Regionen. Mittels der innovativen Methodik werden deutsche Landkreise im Zeitraum 2000 bis 2014 verglichen. Dabei kommt eine robuste nichtparametrische Effizienzanalyse zum Einsatz, deren Ergebnisse durch qualitative Experteninterviews in ausgewählten Kreisen Niedersachsens validiert werden. Die Ergebnisse der Untersuchung zeigen große regionale Unterschiede in der Ausbaueffizienz sowohl zwischen Landkreisen als auch zwischen (Landkreisen in verschiedenen) Bundesländern. Auch bezüglich der Art der drei betrachteten regenerativen Energieträger unterscheiden sich die deutschen Landkreise erheblich. Die empirischen Befunde sind räumlich und zeitlich sehr differenziert und erlauben auch deshalb diverse lokal- und landespolitische Schlussfolgerungen. Insbesondere sollten Landkreise (und Bundesländer) mit bislang eher geringer Ausbaueffizienz von solchen mit hoher Ausbaueffizienz lernen. In der Breite können diese politischen Lerneffekte nachhaltig zum Gelingen der Energiewende beitragen.

Schlüsselwörter: Erneuerbare Energien, Messung, Effizienz, Ausbaupotenzial, Regionen, Deutschland

\footnotetext{
*Corresponding author: Prof. Dr. Rolf Sternberg, Leibniz Universität Hannover, Institut für Wirtschafts- und Kulturgeographie, Schneiderberg 50, 30167 Hannover, Deutschland, E-mail: sternberg@wigeo.uni-hannover.de, ORCID: 0000-0002-7649-0419

Tatjana Bennat, Leibniz Universität Hannover, Institut für Wirtschafts- und Kulturgeographie, Schneiderberg 50, 30167 Hannover, Deutschland, E-Mail: bennat@wigeo.uni-hannover.de

Associate Professor Dr. Tom Broekel, Utrecht University, Department of Human Geography and Spatial Planning, Princetonlaan 8A 3584 CB Utrecht, The Netherlands, E-Mail: t.broekel@uu.nl, ORCID: 0000-0003-4516-4626
} 
Abstract: This paper answers two rarely considered questions: How well do German regions exploit their potential to produce renewable energy and which factors impact on this exploitation efficiency? By applying the new quantitative-empirical concept of exploitation efficiency, we measure the degree, to which regions have exploited their natural and socio-economic potentials of producing energy from renewable source at a specific point in time. This approach allows, with respect to wind power, solar power and biogas energy, a relative comparison of regions, monitoring their performance over time as well as the identification of best-practice regions. Applying our innovative method, we compare German districts in the time period 2000-2014. We use a robust, non-parametric efficiency analysis and validate its results by qualitative expert interviews in selected counties in Lower Saxony. The results show strong disparities in terms of the exploitation efficiency between districts and federal states. This exploitation efficiency moreover varies significantly for different types of renewable energy. We also observe specialization tendencies in this context. Our empirical results are very detailed both from a spatial and from a temporal perspective and therefore allow for drawing several conclusions for local and federal state policies. For instance, those districts (and federal states) with currently rather low exploitation efficiencies need to learn from those with high efficiencies. Such learning effects may sustainably contribute to a successful turnaround in energy policy.

Keywords: Renewable energies, Measurement, Efficiency, Potential for expansion, Regions, Germany

\section{Einleitung}

In Zeiten des globalen Klimawandels wird nachhaltige Energieproduktion zu einem zentralen Thema von Wissenschaft und Politik. Deutschland implementierte auch deshalb im Jahre 2000 das Erneuerbare-EnergienGesetz $\left(E E G^{1}\right)$, das eine nachhaltige Entwicklung der Energieversorgung garantieren soll. Ein Ziel des EEG ist es, bis zum Jahr 2025 mindestens $40 \%$ und bis 2050 mindestens $80 \%$ der Bruttostromerzeugung durch erneuerbare Energien zu gewährleisten ( $\$ 1$ Abs. 2 EEG). 2017 betrug der Anteil erneuerbarer Energien an der Bruttostromerzeugung $36 \%$, wobei mit $49 \%$ der größte Anteil der regenerativen Stromerzeugung auf die Windkraft entfällt (BMWI 2018: 7, 11).

Durch die im EEG angelegte Dezentralisierung der ,grünen' Stromgewinnung gewinnen subnationale Regionen als Handlungsebene an politischer Relevanz, denn Kenntnisse über kleinräumige Potenziale sowie die Vernetzung von regionalen Akteuren erleichtern es, Entwicklungspotenziale zu identifizieren und zu mobilisieren. Der lokalen Ebene kommt somit eine große Bedeutung bei der Energiewende zu (vgl. Keppler 2007). Zudem variiert die Intensität der Energiegewinnung stark zwischen Deutschlands Regionen (vgl. Rauner/Eichhorn/Thrän 2016).

Zur besseren Steuerung der Energiewende bedarf es einer regelmäßigen Überprüfung der Ausbaustände erneuerbarer Energien auf eben dieser Ebene. Der schlichte Vergleich von installierten Leistungen allein ignoriert, dass Regionen unterschiedlich hohe Potenziale in Bezug auf die Nutzung erneuerbarer Energien haben. Um eine faire Bewertung von Regionen zu ermöglichen, sollten die regionalen Potenziale in Beziehung zum erreichten Ausbaustand einer Region gesetzt werden, was in diesem Beitrag als Ausbaueffizienz konzeptualisiert wird. Der interregionale Vergleich dieser Ausbaueffizienz kann als Grundlage für umweltpolitische Strategien sowie für interregionale Kooperationen genutzt werden. Ziel dieses Beitrags ist es daher zu messen, inwieweit Regionen ihre zu einem bestimmten Zeitpunkt bestehenden Potenziale zur Nutzung erneuerbarer Energien ausgeschöpft haben.

Der Beitrag ist wie folgt aufgebaut: Kapitel 2 nähert sich der Konzeption der Ausbaueffizienz zunächst theoretisch und diskutiert den methodischen und empirischen Forschungsstand, bevor das Konzept der Ausbaueffizienz präzisiert wird. Anschließend beschreibt Kapitel 3 eine empirische Methode zur Messung dieser Effizienz, die Data Envelopment Analysis, kurz DEA, sowie deren Bewertung mittels eines Triangulationsansatzes durch qualitative Einzelfallanalysen. Kapitel 4 stellt die empirischen Ergebnisse vor, die in Kapitel 5 kritisch bewertet, zusammengefasst und hinsichtlich ihrer umweltpolitischen Implikationen beurteilt werden.

1 Gesetz für den Ausbau erneuerbarer Energien (ErneuerbareEnergien-Gesetz), ursprüngliche Fassung vom 29. März 2000, zuletzt geändert durch Artikel 1 des Gesetzes vom 17. Dezember 2018. 


\section{Konzeptionelle und methodische Grundlagen}

\subsection{Wind-, Photovoltaik- und Bioenergie als wesentliche regenerative Energien in Deutschland}

Mit der Umwandlung von Bewegungsenergie des Windes in elektrischen Strom stellt die Windkraft den bislang größten Stromlieferanten unter den erneuerbaren Energieträgern dar. Windräder können sowohl an Land (Onshore) als auch auf See (Offshore) installiert werden, wobei das Potenzial an Land durch Freiflächenverfügbarkeit, Mindestabstände zu Ortschaften oder die Rauigkeit einer Region, die die Strukturierung der Oberfläche beschreibt, reduziert wird. Relief und Bewuchs tragen in süddeutschen Regionen zu einer höheren Bremswirkung des Windes bei als im flachen Norden (vgl. Arbach/Gerlach/Kühn et al. 2013). Trotzdem sind schätzungsweise 13 Prozent der Fläche Deutschlands für Windkraftanlagen nutzbar (Lütkehus/Salecker/Adlunger 2013: 2), wobei 95 Prozent der bisher installierten Anlagen auf landwirtschaftlichen Flächen stehen (Rohrig 2014: 36). Ein bedeutender Aspekt beim Thema Flächenverbrauch ist seit einigen Jahren das Repowering, das heißt der Austausch älterer Anlagen gegen jüngere und leistungsstärkere. Dadurch kann der Flächenbedarf von Windkraftanlagen verringert werden (vgl. BMWI 2018).

Neben der Windenergie ist die Photovoltaiktechnologie Zugpferd der Energiewende. In Solarzellen wird die Strahlungsenergie der Sonne in Strom umgewandelt (vgl. BMWI 2018). Sie werden meist auf Dachflächen oder Freiflächen mit einer hohen Sonneneinstrahlung verbaut (vgl. Lödl/Kerber/Witzmann et al. 2010), wodurch in Deutschland potenziell mehr als $400 \mathrm{GW}$ Leistung installiert werden könnten (Quaschning 2016: 33). Der Ausbau auf landwirtschaftlichen Flächen wird momentan nur in wenigen Bundesländern gefördert, die landwirtschaftlich benachteiligte Gebiete entsprechend ausweisen. Der Stromertrag pro Flächeneinheit beträgt jedoch ein Vielfaches des Ertrages aus Energiepflanzen (Günther 2015).

Biomasse ist, ob fest, flüssig oder gasförmig, der zweitgrößte regenerative Stromproduzent in Deutschland (BMWI 2018: 11). Laut der Landwirtschaftskammer Niedersachsen änderten sich die landwirtschaftlichen Anbauverhältnisse durch den Ausbau von Biogasanlagen, wie die Zunahme von Silomais in Gunstregionen für Biogasanlagen zeigt (vgl. Schütte 2012). Zusätzlich werden landwirtschaftliche Reststoffe, wie beispiels- weise Gülle und biogener Abfall von Haushalten, zur Gewinnung von Bioenergie verwendet. ${ }^{2}$

Trotz grundsätzlicher Befürwortung der erneuerbaren Technologien durch Politik und Bevölkerung (vgl. Wunderlich 2012) kommt es auch zu Problemen. Bei der Windenergie und Biogasproduktion entstehen vermehrt Flächennutzungskonflikte. Beispielsweise führte die Erhöhung im Silomaisanbau zu einer Verringerung des Getreideanbaus und zu einer Zunahme des Grünlandumbruches zu Ackerland (Schütte 2012: o. S.). Windkraftanlagen können durch ihren landschaftsästhetischen Effekt den Tourismus beeinflussen (Broekel/ Alfken 2015). Das „Not-In-My-Backyard-Phänomen” (NIMBY) beschreibt, dass Personen zwar den generellen Ausbau erneuerbarer Energien unterstützen, jedoch ihren Ausbau in ihrer unmittelbaren Umgebung behindern (vgl. Kontogianni/Tourkolias/Skourtos et al. 2014). Angst vor gesundheitlichen Nachteilen, negativen Effekten für die lokale Umwelt oder vor Wertverlust des individuellen Eigentums sind wissenschaftlich belegte Ursachen des NIMBY-Effektes (Jami/Walsh 2014). Dieses Phänomen tritt häufig bei Großprojekten wie Solarparks oder Windenergieanlagen auf, kann aber durch Partizipation der Bürger reduziert werden. Auch die Neuregelung der Gewerbesteuerzerlegung im Januar 2009 scheint das Problem verringert zu haben. So bedeutet die Neuregelung für Bürgerwindparks, die vor Ort verwaltet werden, einen Gewerbesteuerzufluss von 100 Prozent (vgl. Bundesverband Windenergie 2013).

Während Photovoltaik-Anlagen eher durch Privatpersonen aufgebaut werden, handelt es sich bei den Betreibern von Biogasanlagen oder Windkraftanlagen häufiger um Bürgerenergiegenossenschaften oder Unternehmen. ${ }^{3}$ Gerade bei diesen können regionale Akteurgruppen aus Verwaltung und Planung, wie beispielsweise Wirtschaftsförderung oder Klimaagenturen, aber auch zivilgesellschaftliche Vereinigungen, wie sie bei Bürgerwindparks, Energiegenossenschaften oder Interessengemeinschaften zu finden sind, entscheidenden Einfluss auf die regionalen Ausbaustände nehmen.

Einen maßgeblichen Einfluss auf die Wirtschaftlichkeit solcher Energieerzeugungsanlagen hatte das erwähnte EEG. Seit dem Inkrafttreten 2000 wuchs der Anteil erneuerbarer Energien an der Stromproduktion stark, denn durch eine garantierte Einspeisevergütung und Anschlusspflicht entstand auch für Privatpersonen

2 Vgl. https://www.umweltbundesamt.de/themen/klima-energie/ erneuerbare-energien/bioenergie (08.04.2019).

3 Vgl. https://www.solarwirtschaft.de/geschaeftsmodelle-pv.html (08.04.2019). 
Investitionssicherheit. Das EEG wurde seit 2000 bereits sieben Mal novelliert, wobei Förderhöhen, Boni-Systeme oder Meldepflichten verändert wurden, ${ }^{4}$ was einen messbaren Einfluss auf den Ausbau der erneuerbaren Energien hatte. Beispielsweise führte die Erhöhung der Vergütung 2004 zu einem stärkeren Ausbau von Biogasanlagen, der nach der Novellierung 2012 wieder nachließ (vgl. Witt/Thrän/Rensberg et al. 2012).

Das EEG ist ein Steuerungselement der nationalen Energiepolitik. Aktuelle Forschungsergebnisse zeigen aber, dass regionale Unterschiede im Ausbau erneuerbarer Energien bestehen (Broekel/Alfken 2015; Diekmann/Schill/Püttner et al. 2017). Da das schnelle und kostengünstige Gelingen der Energiewende von großem politischem Interesse ist (vgl. Weimann 2012), können die Messung dieser Differenzen und die Orientierung an Best-practice-Regionen das Gelingen der Energiewende unterstützen.

\subsection{Der Ausbau der erneuerbaren Energien auf regionaler Ebene}

Die Europäische Union wertet regelmäßig Daten zum Ausbau der erneuerbaren Energien ihrer Mitgliedsstaaten aus, um das Erreichen der Ziele der Europe2020-Strategy (European Commission 2010) für intelligentes, nachhaltiges und gesamtheitliches Wachstum zu überwachen. Im Jahr 2017 führte Schweden dieses Zielerreichungsranking an, gefolgt von Finnland und Lettland. ${ }^{5}$ Länderunterschiede im gesamten Ranking wurden mit natürlichen und klimatischen Begebenheiten erklärt. Beispielsweise entfielen auf Malta drei Fünftel der erneuerbaren Energie auf die Solarenergie, während in Österreich ein Drittel durch Wasserkraft gewonnen wurde. Das Potenzial der einzelnen Energieträger unterscheidet sich signifikant zwischen (nationalen wie regionalen) Räumen, was bisher jedoch bei der Bewertung des Ausbaus vernachlässigt wird. Zudem ist die Datenanalyse auf die nationale Ebene der Mitgliedsstaaten begrenzt. Nationale Analysen, die kleinräumige Unterschiede aufzeigen, wurden durch die EU bisher nicht durchgeführt.

$4 \quad$ Vgl. https://www.unendlich-viel-energie.de/themen/politik/ erneuerbare-energien-gesetz-eeg/erfolgsgeschichte-eeg-daserneuerbare-energien-gesetz (09.04.2019).

$5 \mathrm{Vgl}$. https://ec.europa.eu/eurostat/statistics-explained/images/b/ b4/Share_of_energy_from_renewable_sources_2017_infograph. png (18.06.2019).
Diese Forschungslücke greift das Deutsche Institut für Wirtschaftsforschung in Kooperation mit der Agentur für Erneuerbare Energien und dem Zentrum für Sonnenenergie und Wasserstoff-Forschung Baden-Württemberg auf. Sie vergleichen seit 2008 die Ausbaustände der deutschen Bundesländer unter Berücksichtigung der dortigen politischen Anstrengungen und Erfolge mithilfe eines ständig weiterentwickelten Indikatorensystems. Dessen Hauptbestandteile bilden der bisherige Ausbau, die Ziele und das politische Engagement sowie die Systemintegration erneuerbarer Energien (vgl. Diekmann/ Schill/Püttner et al. 2017). Die Institute bewerten in qualitativer Form die Ziele und Maßnahmen der Bundesländer zur Nutzung erneuerbarer Energien sowie entsprechende Maßnahmen aus den Bereichen Forschung, Bildung und der Ansiedlung von Unternehmen. Zusätzlich werden der Anteil erneuerbarer Energien am Energieverbrauch, die Anlagenkapazitäten und Kennzahlen über Unternehmen im Energiesektor, Beschäftigte, Umsätze, der Aufbau von Infrastruktureinrichtungen und Patentanmeldungen einbezogen. Die empirischen Befunde zeigen Baden-Württemberg, gefolgt von Mecklenburg-Vorpommern und Bayern, auf den vorderen und Hessen, Berlin und das Saarland auf den hinteren Rangplätzen (Diekmann/Schill/Püttner et al. 2017: 112).

Diese Studie betont die Wirksamkeit politischen Eingreifens und die Relevanz einer place-based Regionalpolitik bei der Energiewende, was mit dem im Grundgesetz Deutschlands beschriebenen Subsidiaritätsprinzip kompatibel ist: Regionale Wirtschaftspolitik ist gemeinsame Aufgabe von Kommunen und Ländern (und nicht des Bundes), da sie durch ihre Orts- und Situationskenntnisse die regionale Entwicklung effektiver unterstützen können. Zudem hängt der Ausbau regenerativer Energien in einer Region von regionalen ,Pionieren' ab, die Projekte anleiten und Überzeugungsarbeit leisten (Keppler 2007). Weitere Einflussfaktoren sind nach Keppler (2007) die aktuelle Stimmungslage, die Diffusion von Wissen und die Festlegung von Leitbildern als Orientierungsrahmen. Allerdings stellt sich die Frage, ob die Bundesländer wirklich die geeignete räumliche Ebene sind, um den Ansprüchen einer ortsbezogenen (place-based) Politik und inrer Bewertung gerecht zu werden. Zudem existieren auch innerhalb der Bundesländer große topographische und sozioökonomische Unterschiede, die das Potenzial zum Ausbau erneuerbarer Energien determinieren. Solche Unterschiede werden in existierenden Studien nicht berücksichtigt. Es wird demnach impliziert, dass die geographischen Eigenschaften wie die Verfügbarkeit von (Frei-)Flächen, die Windhöffigkeit, oder der Anfall von Biomasse inner- 
halb der Vergleichsregionen (EU-Mitgliedsstaaten, Bundesländer) gleich verteilt sei.

Unterschiede des räumlichen Potenzials der einzelnen erneuerbaren Energieträger können aber erheblich sein, wie Hoogwijk (2004) in ihrer Bewertung der momentanen und zukünftigen erneuerbaren Energieressourcen zeigt. Zwar nimmt diese Studie eine globale Perspektive ein und analysiert Unterschiede zwischen Weltregionen, dennoch lassen sich die grundsätzlichen Erkenntnisse auch auf kleinräumigere Gebiete übertragen. Hoogwijk (2004: 43 ff.) erstellt ein System zur globalen Potenzialbewertung von Wind-, Photovoltaik- und Bioenergie und unterscheidet zwischen theoretischem Potenzial (theoretische Ressourcenverfügbarkeit, z. B. Globalstrahlung), geographischem Potenzial (Nutzbarkeit von Flächen zur Energiegewinnung), technischem und wirtschaftlichem Potenzial (Kosten der Energieträger) sowie Implementierungspotenzial in das Energiesystem (Akzeptanz und politischer Wille). Für die Potenzialbewertung von Bioenergie werden so beispielsweise Grasland- und Landwirtschaftsflächen und die Größe des Viehbestandes sowie die Menge an organischem Abfall betrachtet. Ähnlich wird für die Windenergie vorgegangen: Hauptbewertungsfaktor ist die Summe der Flächen, auf denen Onshore-Anlagen aufgebaut werden können. Grundlegend sind dafür passende Windverhältnisse, ausreichende Abstände zu Wohngebieten, das Fehlen von ausgewiesenen Naturschutzgebieten und keine zu starken Höhenunterschiede. Bei der Potenzialbewertung für die Photovoltaik fallen die technischen und wirtschaftlichen Potenziale stärker ins Gewicht als die geographischen Gegebenheiten, da sich die Strahlungsintensität kleinräumig kaum unterscheidet.

Die von Hoogwijk (2004) identifizierten Potenziale variieren geographisch erheblich: Für Windkraft sind Kanada, die USA und Südamerika besonders geeignet. Niedrige Kosten für die Installation von PhotovoltaikAnlagen ergeben ein hohes Potenzial in Afrika, Ozeanien und Südostasien. Aufgrund großer potenzieller Anbauflächen für Bioenergiepflanzen werden Russland, Afrika und die USA als Gunstregionen für Biogas ausgewiesen. Ein Blick auf die Unterschiede in der Windhöffigkeit, auf die Verfügbarkeit von Agrarflächen und das topographische Profil innerhalb Deutschlands verdeutlicht allerdings, dass ähnliche (wenn auch in ihrer absoluten Höhe sicherlich etwas geringere) Unterschiede auch hierzulande existieren. Entsprechend verfügen subnationale Regionen über sehr verschiedene Potenziale zum Ausbau der erneuerbaren Energien. So zeigt beispielsweise die Studie von Rauner, Eichhorn und Thrän (2016), dass die lokale Energieproduktion in Deutschland erheb- lich variiert, was unter anderem auf Unterschiede in der Flächennutzung und Flächennutzungseignung zurückzuführen ist.

Ein bewertender Vergleich des Ausbauerfolges von erneuerbaren Energien in Deutschland, der diese Potenzialunterschiede nicht berücksichtigt, wird somit im besten Fall zu wenig informativen, im schlimmsten Fall zu stark verfälschten Ergebnissen führen, die wiederum die falschen Signale an nationale und regionale Entscheider senden.

Der knappe Überblick zum Forschungsstand zeigt, dass es erstens keine wissenschaftlichen Arbeiten zur Ausschöpfung des Potenzials an regenerativen Energien für Deutschlands Regionen gibt und dass, zweitens, eine Übertragung existenter Studien für die globale Ebene auf deutsche Regionen machbar ist und auf energiepolitisches Interesse stoßen sollte. Die skizzierte Forschungslücke soll mit dem vorliegenden Beitrag geschlossen werden. Er stellt einen Vergleich des Ausbauerfolges von deutschen Regionen in Bezug auf die erneuerbaren Energien vor, bei dem explizit die Potenziale zum Ausbau Berücksichtigung finden. Es geht also um die Frage, inwieweit Regionen ihre zu einem Zeitpunkt bestehenden, technisch nutzbaren Potenziale zur Nutzung erneuerbarer Energien ausgeschöpft haben.

\section{Methodisches Vorgehen}

\subsection{Daten}

Als regionale Ebene werden für den deutschlandweiten Vergleich Landkreise gewählt, da auf dieser Ebene genügend politische Independenz vorhanden und gleichzeitig eine gute Datengrundlage verfügbar ist. Für die Auswahl der Regionen ist es wichtig, dass nur strukturell ähnliche Raumeinheiten miteinander verglichen werden (vgl. Scheel 2000). Da in städtischen Regionen der Ausbau von Biogas- und Windkraftanlagen nahezu ausgeschlossen ist, bleiben die 107 kreisfreien Städte sowie die Kommunalverbände Region Hannover und Städteregion Aachen in der empirischen Analyse unberücksichtigt. Der Beitrag konzentriert sich entsprechend auf den Ausbau der erneuerbaren Energien in den 290 deutschen Landkreisen.

Die in dieser Untersuchung verwendeten Daten stammen von Statistischen Ämtern des Bundes und der Länder, dem Deutschen Wetterdienst und EnergyMap. ${ }^{6}$

6 http://www.energymap.info/ (14.06.2019). 
Aufgrund der Datenlagen betrachten wir grundsätzlich den Zeitraum 2000 bis 2014. Leider liegen keine aktuelleren Daten vor.

Auf diesen Daten aufbauend wird der Ausbaustand der erneuerbaren Energien über die installierte Nennleistung in Kilowatt gemessen. Von der reinen Anzahl der Anlagen als Output-Variable wird abgesehen, da beispielsweise Repowering-Maßnahmen bei Windenergie zu einer Steigerung der Produktionskapazität führen, jedoch nicht zu einer Zunahme der Zahl der installierten Anlagen. Gleiches gilt für die Größenunterschiede von Photovoltaik-Anlagen auf Dachflächen im Gegensatz zu Freiflächenanlagen. Des Weiteren werden OffshoreAnlagen aus der Analyse ausgeschlossen, da sie sich nicht eindeutig einer Region zuweisen lassen. Zudem können aufgrund der Datenlage nur Anlagen berücksichtigt werden, die durch das EEG gefördert werden. Kraftstoff- und Wärmeerzeugung bleiben daher außen vor. Der Fokus liegt auf der Stromerzeugung. Weiterhin bleiben die Energieträger Wasserkraft und Hausmüll unberücksichtigt, da hier erhebliche Datenlücken bestehen. Entsprechend wird der Ausbaustand der erneuerbaren Energien zur Stromerzeugung einer Region durch die installierte Nennleistung der Photovoltaik-Anlagen, Windkraftanlagen und Biogasanlagen beschrieben.

Die Studie von Hoogwijk (2004) verdeutlicht, dass für die verschiedenen Träger erneuerbarer Energien unterschiedliche Voraussetzungen gegeben sein müssen, bzw. dass ihre ökonomische Attraktivität durch unterschiedliche sozioökonomische und geographische Faktoren beeinflusst wird. Nachfolgend werden für jeden der drei im vorliegenden Beitrag berücksichtigten Energieträger die Faktoren oder Determinanten diskutiert, die in der späteren Effizienzanalyse Verwendung finden. In Tabelle 1 sind sie zusammenfassend aufgelistet.

Windkraftanlagen werden vornehmlich auf landwirtschaftlichen Flächen aufgestellt. Daher geht dieser Faktor als Flächenpotenzial in die Berechnung ein. Zusätzlich spielt die Windkraftnutzungseignung, die durch den durchschnittlichen jährlichen Ertrag einer Referenzwindkraftanlage anhand der durchschnittlichen Windstärke der Periode 1981 bis 2000 definiert wird, eine Rolle. Auch die durchschnittliche Hangneigung wird berücksichtigt, da sonst Gebiete mit starkem Gefälle gleichgestellt würden mit Gebieten im Flachland. Die Errichtung von Windkraftanlagen setzt jedoch relativ ebenerdige Flächen voraus.

Bei der Photovoltaik stellt die Variable "Gebäudeund Freifläche" einer Region einen der wichtigsten regionalen Potenzialfaktoren dar, da Photovoltaik-Anlagen oftmals auf Gebäuden oder in Form von Anlagenparks auf Freiflächen installiert werden. Die Landwirtschaftsfläche taugt nicht als Input-Variable, da die Flächen zwar grundsätzlich für Installationen geeignet sind, jedoch nur in einigen wenigen Bundesländern überhaupt für Photovoltaik-Anlagen genutzt werden dürfen. Ebenfalls muss die Bevölkerungszahl berücksichtigt werden. Sie spiegelt einerseits die Möglichkeit für den Ausbau auf privaten Eigenheimen wider, andererseits korreliert sie aber auch mit dem Investitionspotenzial eines Landkreises. ${ }^{7}$ Die durchschnittliche Hangneigung spielt auch für die Photovoltaik eine Rolle. Zwar benötigen die Anlagen einen gewissen Einfallwinkel des Sonnenlichtes, dennoch werden sie auf Hängen wegen der erschwerten Zugänglichkeit und der Gefahr von Bodenerosion nur selten installiert (Franke 2014). Entgegen der Intuition wird die Globalstrahlung nicht einbezogen, da sie in Deutschland kaum variiert (vgl. Hoogwijk 2004). Linder (2013: 92) zeigt zudem, dass die Globalstrahlung den Ausbau von Photovoltaik-Anlagen nicht beeinflusst, sondern eher lokale Faktoren wie Dachausrichtung oder Dachverschattung ausschlaggebend sind. Für Letztere gibt es leider keine flächendeckenden Daten.

Beim Biogas ist die vorhandene Landwirtschaftsfläche ausschlaggebend für die Produktion von Biomasse wie beispielsweise Silomais. Obwohl die Kulturpflanze 40-75 Prozent des Ausgangsstoffes für die Biogasproduktion liefert, ${ }^{8}$ wird ihr Anbau im vorliegenden Beitrag nicht berücksichtigt. Dies hat mehrere Gründe. Die Daten zur Anbaufläche auf Kreisebene liegen nur für das Jahr 2007 vor. Deutschlandweite Messungen ergaben jedoch eine zunächst starke und in den letzten Jahren (2016-2018) noch leichte Steigerung des Silomaisanbaus. ${ }^{9}$ Das bedeutet, dass diese Zahlen nicht auf die untersuchten Zeitperioden übertragbar sind. Zudem ist die Anbaufläche für Silomais relativ leicht beeinflussbar, was der Idee von regionalen Potenzialen für den Ausbau erneuerbarer Energien widerspricht. Schließlich ist die Variable Silomais ebenfalls indirekt in der Variable Landwirtschaftsfläche enthalten (beide korrelieren signifikant und positiv mit $r=0,8$ ). Als weitere wichtige Einflussgröße muss der biogene Abfall der Haushalte sowie Gülle aus landwirtschaftlicher Nutztierhaltung in die Analyse einbezogen werden. Dies erfolgt über die Variablen Bevöl-

7 Tabelle A im online supplement.

8 Vgl. http://www.Ifl.bayern.de/ipz/mais/026660/index.php (09.04.2019) und http://www.renewable-energy-concepts.com/ german/bioenergie/biogas-basiswissen/biogaszusammensetzung. html (09.04.2019).

9 https://www.destatis.de/DE/Themen/Branchen-Unternehmen/ Landwirtschaft-Forstwirtschaft-Fischerei/Feldfruechte-Gruenland/ Tabellen/liste-feldfruechte-zeitreihe.html (09.04.2019). 
kerungszahl und Viehbestand, deren Zunahmen auch das Biomassepotenzial eines Landkreises erhöhen (vgl. Hoogwijk 2004). Wie bei den beiden erstgenannten Energieträgern spielt auch hier die durchschnittliche Hangneigung eine gewisse Rolle, da Biogasanlagen bei steilem Gefälle nicht gebaut werden können bzw. die Anlieferung der Rohstoffe deutlich aufwendiger ist.

\subsection{Quantitative Analyse}

\subsubsection{Motivation des empirischen Ansatzes}

Um den Ausbaustand von Regionen unter Berücksichtigung ihrer Potenziale zur Erzeugung erneuerbarer Energien zu bewerten, greifen wir auf eine Analogie zum Produktionsprozess zurück. Wir fassen Kreise als Einheiten auf, die ihren Input (ihr Potenzial zum Ausbau von erneuerbaren Energien) maximal ausschöpfen wollen, um einen möglichst großen Output (erzeugte erneuerbare Energie) zu erreichen. Verschiedene Faktoren, z. B. (fehlender) politischer Wille, beeinflussen ihre Fähigkeit zur Ausschöpfung jedoch, was zur Varianz beim Ausbauerfolg führt. Wir interpretieren die regionalen Potenziale also als „Inputs" und die installierte Leistung zur Nutzung erneuerbarer Energien als „Outputs“. Entsprechend wird der Ausbauerfolg als Effizienz der Ausschöpfung dieser Potenziale („Ausbaueffizienz“) verstanden. Die empirische Ermittlung und Bewertung dieser Effizienz steht im Mittelpunkt des Beitrages.

Die vorherigen Ausführungen machen deutlich, dass es in jedem Landkreis mehrere Inputs (regionale Faktoren) und mehrere Outputs (spezifisch für jeden Energieträger) gibt, sodass die methodische Herausforderung darin besteht, die Effizienz für ein Multi-Inputund Multi-Output-Szenario empirisch zu ermitteln. Wir greifen dafür auf eine nichtparametrische Effizienzanalyse zurück. Dadurch werden nicht nur die multidimensionalen regionalen Potenziale bei der Bewertung des Ausbaustandes berücksichtigt, sondern gleichzeitig die Probleme der Größeneffekte und der Gewichtung der unterschiedlichen Faktoren modellendogen gelöst. Das ermöglicht eine fairere Bewertung von Regionen, das Aufdecken von Unterschieden beim Ausbauerfolg, die Identifizierung von noch bestehenden Ausbaupotenzialen sowie die Identifikation von Best-practice-Regionen. Letztere repräsentieren Fallbeispiele, von denen bislang weniger erfolgreiche Regionen lernen können. Die so quantitativ-empirisch ermittelten Ergebnisse werden anschließend mittels einer qualitativen, nachgelagerten Analyse validiert.
Tabelle 1: Determinanten (Input-Variablen) der installierten Nennleistung je Energieträger

\begin{tabular}{ll}
\hline Energieträger & Input \\
\hline Windkraft & $\begin{array}{l}\text { landwirtschaftliche Flächen } \\
\text { Windkraftnutzungseignung } \\
\text { durchschnittliche Hangneigung } \\
\text { Gebäude- und Freifläche } \\
\text { Bevölkerungszahl } \\
\text { durchschnittliche Hangneigung } \\
\text { Landwirtschaftsfläche } \\
\text { Bevölkerungszahl } \\
\text { Viehbestand } \\
\text { durchschnittliche Hangneigung }\end{array}$ \\
\hline
\end{tabular}

\subsubsection{Quantitativ-empirische Analyse}

Für die quantitativ-empirische Ermittlung der Ausbaueffizienz greifen wir auf eine Data Envelopment Analysis (DEA) zurück, die für multiple Input-/Output-Fälle ein radiales Effizienzmaß berechnet (vgl. Padberg/Werner 2005). Diese Methodik wird häufig im öffentlichen bzw. Nonprofit-Sektor angewandt, das heißt in Situationen, in denen Kosten und Preise im Regelfall unbekannt sind (vgl. Scheel 2000). Die Data Envelopment Analysis ist ein nichtparametrisches Verfahren zur Effizienzmessung von Einheiten (Decision Making Units (DMU) = Regionen), die zur Erstellung von Outputs (Ausbaustand erneuerbarer Energiegewinnung) bestimmte Input-Faktoren (hier: regionales Potenzial zum Ausbau erneuerbarer Energien) benötigen. Eine Decision Making Unit gilt dann als effizient, wenn es ihr im Vergleich zu den übrigen Regionen gelingt, bei gegebenem Input möglichst viel Output zu erzeugen (Output-Orientierung; vgl. Scheel 2000).

Unter der Annahme freier Verschwendbarkeit und Konvexität der Bewertungskriterien entwickelt die Data Envelopment Analysis eine Technologiemenge, die alle möglichen Input-Output-Kombinationen umfasst. Der Effizienzwert einer Decision Making Unit wird durch den Abstand seines Input-Output-Vektors zum effizienten Rand der Technologiemenge durch lineare Programmierung berechnet. Der effiziente Rand besteht aus einer Teilmenge der Technologien, die Pareto-Koopmanseffizient sind (vgl. Hammerschmidt/Wilken/Staat 2009). Somit liegen auf dem Rand der Produktionsfunktion alle effizienten (best practice) Decision Making Units, die alle ineffizienten Decision Making Units ,einhüllen‘. 
Die verwendete Spezifikation ist ein outputorientiertes Modell mit variablen Skalenerträgen von Banker, Charnes und Cooper (1984). Verglichen mit der Annahme konstanter Skalenerträge ist die Annahme variabler Skalenerträge weniger restriktiv (vgl. Kerpen 2016). Die Output-Orientierung wird gewählt, da es das Ziel ist zu ermitteln, welche Regionen - gegeben ihr Potenzial (Input) - ihren Ausbaustand (Output) (noch) nicht maximiert haben (vgl. Daraio/Simar 2007).

Im DEA-Modell können die verschiedenen Inputs bzw. Outputs in unterschiedlichen Skalen vorliegen, solange sie metrischer Natur und nicht negativ sind. Ihre Gewichtung und die Entwicklung der Frontierfunktion erfolgt modellendogen (vgl. Backhaus/Wilken 2003), sodass für jede Decision Making Unit kein besseres Gewichtungsschema existiert. Das bedeutet, für jede Region werden die verschiedenen Input-Faktoren (Dimensionen, in denen das Ausbaupotenzial beschrieben wird) und Output-Faktoren (erneuerbare Energieträger) so gewichtet, dass für jede Region kein höherer Effizienzwert möglich ist. Anders ausgedrückt: Die Data Envelopment Analysis umgeht das sonst übliche Problem der Faktorengewichtung bei einer Bildung von Indizes und bewertet jede Region auf eine für sie optimale Art und Weise.

Technisch wird beim Leistungsvergleich einer DMU0 mit allen anderen DMU der Quotient aus den Outputs und Inputs (e) unter mehreren minimiert, unter der Nebenbedingung, dass der Effizienzwert im Intervall $[1, \infty)$ liegt, wobei $e=1$ das Best-practice-Niveau darstellt (vgl. Cantner/Krüger/Hanusch 2007). Werte über 1 kennzeichnen Ineffizienzen. Beispielsweise lässt sich $\mathrm{e}=2$ so interpretieren, dass die Decision Making Unit mit gegebenem, aber effizienterem Einsatz ihrer Inputs einen Output von 200 Prozent ihres tatsächlichen Outputs hätte erreichen können. Demnach ergibt sich das Minimierungsproblem für die a-te der $\mathrm{n}$ Decision Making Units:

$$
\min _{u, v} e_{a}^{\text {out }}=\frac{\sum_{i=1}^{p} v_{a, i} * x_{a, i}-v_{a}^{*}}{\sum_{j=1}^{q} u_{a, j} * y_{a, j}}
$$

unter den Nebenbedingungen

$$
\begin{gathered}
\frac{\sum_{i=1}^{p} v_{a, i} * x_{k, i}-v_{a}^{*}}{\sum_{j=1}^{q} u_{a, j} * y_{k, j}} \geq 1 \forall k=1, \ldots, n . \\
v>0 \\
u>0
\end{gathered}
$$

Hierbei stellen $x$ die $p$ verschiedenen Input-Faktoren, i und y die q verschiedenen Output-Faktoren j dar; v bzw. $u$ sind ihre entsprechenden endogen ermittelten Gewichtungsfaktoren.

Aufgrund der wenigen und sehr konservativen Annahmen findet die Data Envelopment Analysis in vielen Bereichen Anwendung (vgl. Simar 2007). Allerdings weist sie auch einige Limitationen auf: Erstens sind die Ergebnisse anfällig für Ausreißer (vgl. Hammerschmidt/Wilken/Staat 2009). Zweitens führt ihre deterministische Natur zu Schwierigkeiten, statistische Rückschlüsse zu ziehen. Drittens ergibt sich ein „Fluch der Dimensionen“, der besagt, dass mit steigender Inputund Output-Anzahl auch die Anzahl der als effizient ausgewiesenen Decision Making Units steigt (Dorfard 2014: 33).

Um den genannten Limitationen entgegenzuwirken, kommt im vorliegenden Beitrag ein robusteres Verfahren der Data Envelopment Analysis zur Anwendung. Vorab wird dabei zur Identifizierung von Ausreißern die Methode des Order-m-Schätzers nach Cazals, Florens und Simar (2002) durchgeführt, was zur Ermittlung eines erwarteten maximalen Output-Wertes für jede Decision Making Unit führt. Weist eine Decision Making Unit einen deutlich höheren Wert als diesen Erwartungswert auf, kann es sich um einen Ausreißer handeln und eine Einzelfallüberprüfung sollte folgen (für eine detaillierte Beschreibung des Vorgehens vgl. Daraio/Simar 2007; Hammerschmidt/Wilken/Staat 2009).

Nach der Ausreißeranalyse wird die Sensitivität der Effizienzwerte gegenüber Variationen der geschätzten Frontierfunktion durch bootstrapping analysiert (vgl. Simar/Wilson 1998). Dies hat zwei Effekte. Zum einen wird die DEA-Analyse um ein stochastisches Element erweitert und ist ergo nicht mehr ,deterministisch'. Zum anderen bedeutet die Anwendung von bootstrapping, dass die um potenzielle stochastische Verzerrung korrigierten DEA-Effizienzwerte niemals exakt den Wert 1 einnehmen. Die letzte Limitation, „der Fluch der Dimensionen", spielt in der vorliegenden Analyse aufgrund des günstigen Verhältnisses von empirischen Beobachtungen 290 zu maximal 9 Variablen (Input und Output) keine Rolle.

Die Effizienzanalyse wird in mehreren Spezifikationen durchgeführt: sowohl für den Ausbau der einzelnen Energieträger Biogas-, Photovoltaik- und Windenergie als auch für alle Energieträger insgesamt bis zum Jahr 2014 (aktuellste Daten für den Stand des Ausbaus). Da es außerdem von Interesse ist, ob eine Region bereits lange in den Ausbau erneuerbarer Energieträger investiert oder ob sie erst in jüngerer Vergangenheit ausge- 
baut hat, wird zusätzlich der Ausbau in drei Perioden (2000 bis 2004, 2005 bis 2009, 2010 bis 2014) untersucht. Den Novellierungen des EEG 2004, 2009 und 2014 wird durch die Abgrenzung der Perioden Rechnung getragen. Da diese größtenteils im Spätsommer des jeweiligen Jahres in Kraft traten, werden die entsprechenden Jahre der EEG-Novellierungen der vorherigen Periode zugeordnet.

Weiterhin interessiert uns die Dynamik des Ausbaus, das heißt die Veränderung der Effizienz zwischen den Perioden. Hierfür wird, wie in der Effizienzliteratur üblich, der Malmquist-Index berechnet (vgl. Cantner/Krüger/ Hanusch 2007). Da die Effizienzmessung der Periode 1 relativ zur Frontierfunktion (Menge an effizienten Regionen) der Periode 1 und die Effizienzmessung der Periode 2 relativ zur Frontierfunktion der Periode 2 erfolgt, kann über die reinen Effizienzwerte keine Aussage zur Produktionsveränderung erfolgen, da sich auch die Frontierfunktion an sich verschoben haben kann, da die effizienten Regionen gewechselt oder noch effizienter geworden sind. Durch die Verwendung und Möglichkeit der Zerlegung des Malmquist-Index können diese Verschiebungen jedoch berücksichtigt werden, was die Analyse der Effizienzveränderung über die Zeit ermöglicht.

Eine Voraussetzung zur Anwendung der DEA ist eine generell positive Beziehung zwischen Inputs und Outputs (vgl. Scheel 2000). Eine Rangkorrelationsanalyse bestätigt dieses für die hier verwendeten Daten. ${ }^{10}$

\subsubsection{Problemzentrierte Experteninterviews}

Um die Ergebnisse der Effizienzanalyse zu validieren, wurden anschließend problemzentrierte Interviews mit Experten ausgewählter Landkreise durchgeführt. Ziele dieser Interviews sind erstens die Überprüfung der quantitativ-empirischen Ergebnisse auf Plausibilität und zweitens der Erhalt von Hinweisen bezüglich der Erklärungen für die ermittelte interregionale Variation der Effizienz. Wir erwarten insbesondere, dass die Ausbaueffizienz durch regionale Akteurgruppen aus Verwaltung, Planung und zivilgesellschaftlichen Vereinigungen beeinflusst wird. Ihre Handlungslogiken werden dabei durch die Situation in einer Region bestimmt (vgl. Keppler 2007). Dies soll empirisch mit den Interviews überprüft werden.

Die Experten wurden dabei schrittweise nach dem Konzept des "theoretischen Samplings" von Glaser und Strauss (1998) ausgewählt. In einem ersten Schritt

10 Tabelle A im online supplement. wurden die genauer zu untersuchenden Landkreise aufbauend auf den ermittelten Effizienzwerten ausgewählt: Besonders effiziente/ineffiziente, auf einen bestimmten Energieträger spezialisierte oder bezüglich ihrer Effizienzwerte sehr dynamische Landkreise qualifizierten sich als potenzielle Fallstudienregionen. Im zweiten Schritt erfolgte eine Internetrecherche zum Ausbau erneuerbarer Energien in den Fallstudienregionen, in der mögliche Experten identifiziert wurden. Im dritten Schritt wurden teilstrukturierte Interviews mittels eines Gesprächsleitfadens durchgeführt. Inhaltlich beziehen sich die Fragen auf die Validierung der DEA-Ergebnisse und die Gründe für die Effizienzunterschiede. Die Gespräche wurden aufgezeichnet und anschließend transkribiert. Die Transkripte wurden zunächst nach dem Konzept des thematischen Kodierens nach Flick (1991) aufgebrochen. Der verwendete Interviewleitfaden erleichterte die Entwicklung von Kategorien und ermöglichte einen Vergleich der Fälle. Das thematische Kodieren erfolgte in drei Phasen. Zunächst wurden Einzelfallanalysen durchgeführt, in denen die prägnantesten Textstellen im Interview, die auf Besonderheiten oder auch Allgemeingültiges hinweisen, herausgesucht wurden. Anschließend wurde aus den ersten Fällen ein Kategoriensystem entwickelt, das fortlaufend in den nächsten Interviews modifiziert wurde. Schließlich wurden Gemeinsamkeiten, Unterschiede, Beziehungen oder Widersprüche zwischen den Expertenmeinungen herausgearbeitet, auf denen exemplarische Verallgemeinerungen bzw. Typenbildungen basieren (vgl. Miles/Huberman/Saldaña 2014). Um die Anonymität der Interviewpartner zu gewährleisten, erfolgt die Ergebnispräsentation unter Angabe von Codenummern, die den Interviewten in der Reihenfolge der Untersuchung zugeordnet wurden.

\section{Ergebnisse}

\subsection{Ausreißeranalyse}

Bevor die Ergebnisse der nichtparametrischen Effizienzanalyse vorgestellt werden können, muss überprüft werden, ob eventuelle Ausreißerbeobachtungen die Berechnungen verzerren. Da es das Ziel ist, nur die allergrößten Ausreißer zu eliminieren und die Anzahl der Untersuchungsregionen möglichst groß zu halten, wird ein Schwellenwert auf alpha=1,6 festgesetzt. Damit werden neun der 290 Untersuchungsregionen als potenzielle Ausreißer identifiziert (3,1\%), was unterhalb des heuristischen Wertes ausgeschlossener Deci- 
sion Making Units von $\sqrt{ } \mathrm{n} / \mathrm{n}=5,8 \%$ liegt (Daraio/Simar 2007: 81). Bei den potenziellen Ausreißer-Landkreisen Dithmarschen, Nordfriesland und Schleswig-Flensburg handelt es sich um Küstenstandorte mit sehr vielen und großen Windenergieanlagen, die durch den historisch frühen und nachfolgend massiven Ausbau kaum vergleichbar mit anderen Landkreisen sind. Ähnliches gilt für die Landkreise Aurich und Emsland, wo Firmensitz und Produktionsstätten des Windanlagenherstellers Enercon verortet sind. Der Landkreis Stade fällt durch sehr hohe Werte im Bereich Biogas und die Landkreise Borken und Steinfurt durch sehr hohe, mit anderen Regionen nicht vergleichbare Ausbaustände von Photovoltaik auf, weshalb auch sie ausgeschlossen werden. Zudem wird der Landkreis Diepholz ausgeschlossen, der trotz eines sehr geringen Potenzials überraschend hohe Ausbaustände vorzuweisen hat. Auffällig ist, dass alle Ausreißer entweder küstennahe oder hochspezialisierte landwirtschaftliche Gunstregionen sind.

Ähnlich wurde bei der Betrachtung der einzelnen Energieträger vorgegangen: Bei der Windenergie wurde alpha ebenfalls mit 1,6 festgelegt, was zum Ausschluss von sechs Decision Making Units führt. ${ }^{11}$ Wie auch bei der vorherigen Analyse werden Dithmarschen, Nordfriesland und Aurich ausgeschlossen. Hinzu kommen die Landkreise Paderborn, Prignitz und Uckermark, bei denen es sich ebenfalls um landwirtschaftliche Gunstregionen handelt. Bei der Betrachtung der Photovoltaik werden drei Landkreise ausgeschlossen: Borken, Sitz eines Tochterunternehmens von Siemens, das in der Umwelttechnik tätig ist, sowie die Landkreise Passau und Ostallgäu, die sehr hohe Effizienzwerte aufweisen. Bei der Effizienzanalyse der Biogasenergie bleibt lediglich der Landkreis Stade aufgrund seines extremen Effizienzwertes unberücksichtigt. ${ }^{12}$

Insgesamt macht die Ausreißeranalysie deutlich, dass es extrem auf einen einzelnen Energieträger spezialisierte Regionen in Deutschland gibt. Diese (wenigen) Regionen nehmen eine einmalige Stellung ein und eignen sich daher nicht zum Vergleich mit anderen Regionen.

\subsection{Ergebnisse der Effizienzanalyse}

Aufbauend auf den Ausreißeranalysen werden nachfolgend die Ausbaueffizienzwerte zunächst für alle erneuerbaren Energieträger bis 2014, anschließend separat

11 Abbildung A im online supplement.

12 Abbildung A im online supplement. für die Wind-, Solar- und Biogasenergie bis 2014 und abschließend die Veränderungen der Effizienz zwischen den Perioden dargestellt. Die Unterscheidung zwischen einer Gesamt- und Einzelbetrachtung der erneuerbaren Energieträger gibtAuskünfte darüber, ob zwischen einzelnen Energieträgern eine Austauschbeziehung bzw. eine gegenläufige Abhängigkeit besteht, denn unterschiedliche raumstrukturelle Prägungen können bestimmte Energieträger begünstigen, was in der Gesamtbetrachtung nicht auffallen würde. Die ausgewiesenen robusten Effizienzwerte sind so zu interpretieren, dass bei niedrigen Werten nur geringe Steigerungsmöglichkeiten der installierten Leistungen der erneuerbaren Energien bei gegebenen regionalen Potenzialen vorhanden sind.

\subsubsection{Gesamtbetrachtung der Ausbaueffizienz der erneuerbaren Energieträger}

Abbildung 1 zeigt die Ausbaueffizienzen für die Gesamtbetrachtung der erneuerbaren Energieträger bis zum Jahr 2014. Der Median liegt bei 1,29; die Werte streuen nur wenig (Spannweite=2,84). Das bedeutet, dass Regionen im Durchschnitt ihre installierten Leistungen um 30 Prozent steigern müssten, um effizient zu werden. Überdurchschnittlich hohe Effizienzwerte weisen die Landkreise in Baden-Württemberg und Bayern auf, während Sachsen und Thüringen die meisten ineffizienten Landkreise besitzen. Tabelle $\mathrm{B}^{13}$ zeigt die vorderen und hinteren zehn Rangplätze der Landkreise. Die größte Effizienz konnte im Landkreis Rottweil $(1,10)$ gemessen werden, die größte Ineffizienz im IIm-Kreis $(3,94)$.

In der dynamischen Betrachtung werden die Effizienzen zwischen den Perioden 2005-2009 und 20002004 (P2 zu P1) sowie zwischen den Perioden 2010-2014 und 2005-2009 verglichen (P3 zu P2). Wie Abbildung 2 zeigt, kam es in der Periode P2 zu einer Verbesserung der Effizienz in 188 Landkreisen, in 34 Landkreisen zu keiner Veränderung und in den übrigen 59 zu einer Verschlechterung der Effizienz. Eine Verschlechterung ist hierbei relativ zu interpretieren. Im Vergleich zu den Best-practice-Regionen sind diese Kreise zurückgefallen, sie können aber durchaus einen absoluten Zuwachs bei der installierten Leistung realisiert haben.

Der Medianwert der Veränderung beträgt etwa 127. Das bedeutet, dass sich in mindestens $50 \%$ der Kreise die Effizienz um etwa $30 \%$ verbessert. Die stärkste Verbesserung verzeichnet der Landkreis Erding, die

13 Tabelle B im online supplement. 


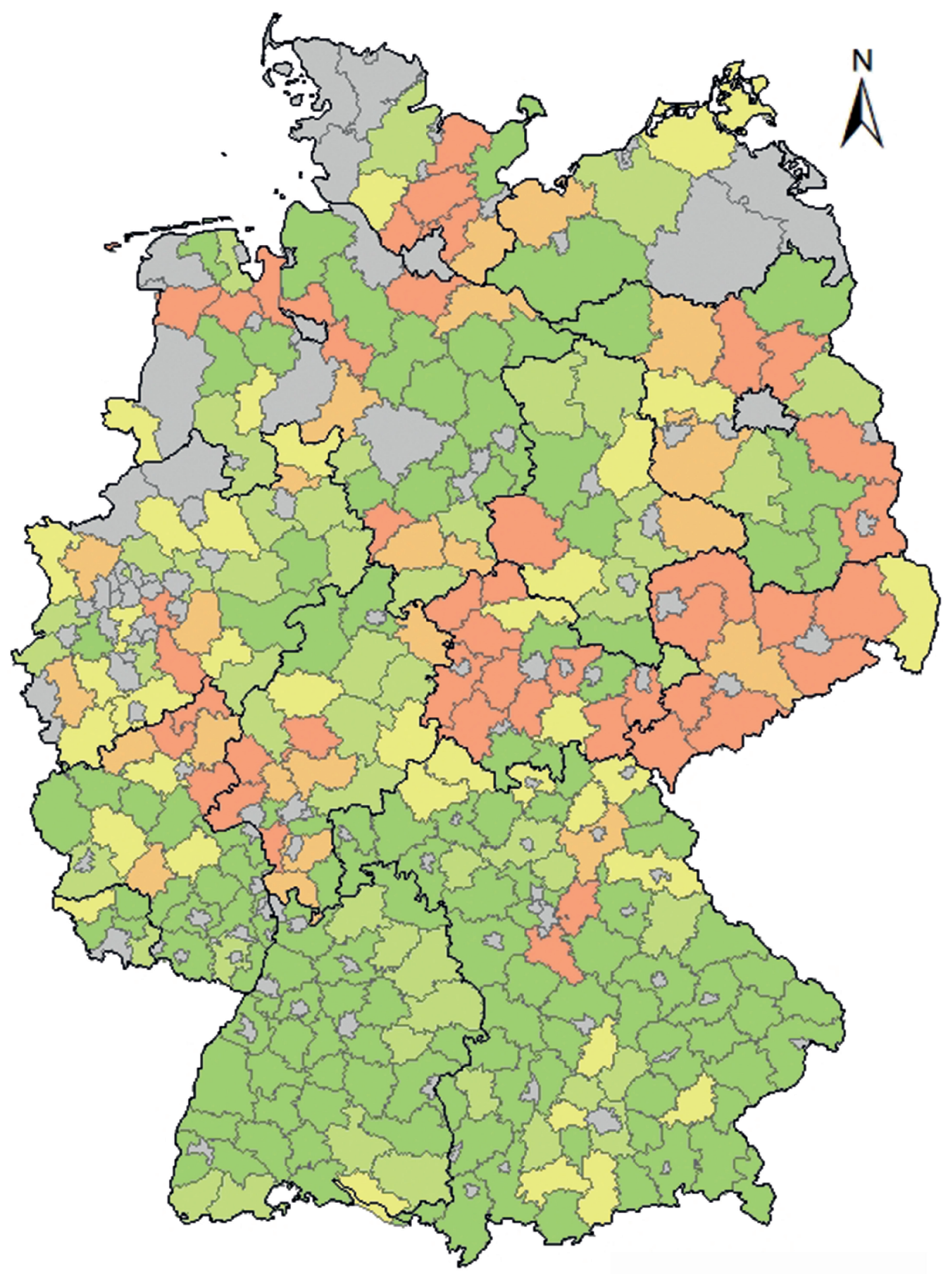

$\square$ nicht betrachtet (121)
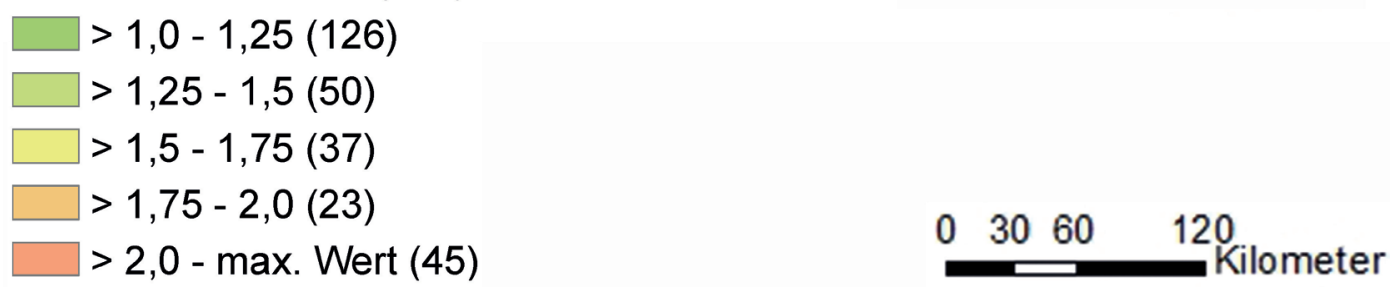

Abbildung 1: Robuste Effizienzwerte der Landkreise beim Ausbau der erneuerbaren Energieträger 2000-2014 insgesamt 


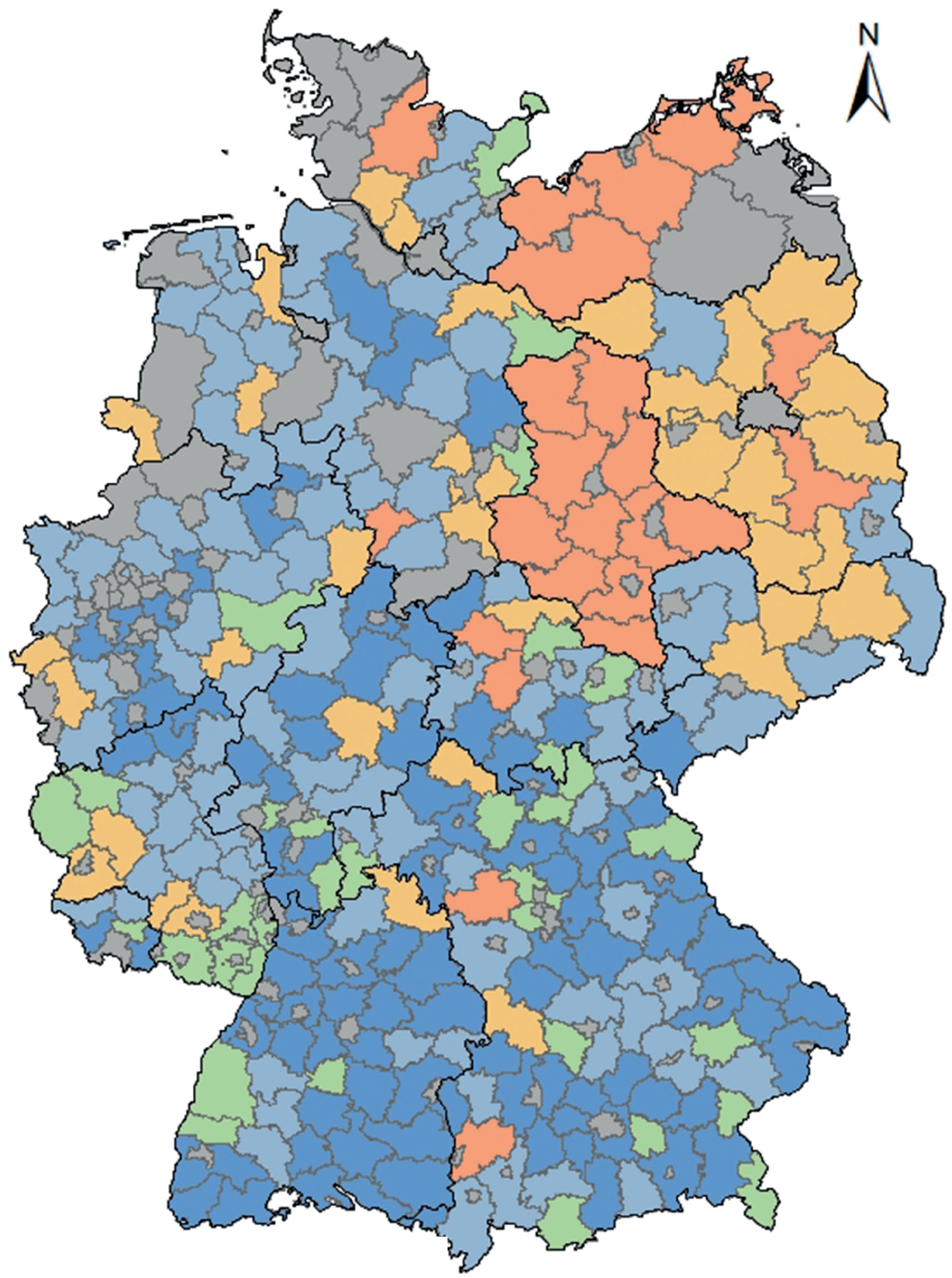

nicht betrachtet (121)

Verschlechterung > $50 \%$ (23)

Verschlechterung $<=50 \%$ (36)

keine Veränderung (34)
Verbesserung <= $500 \%$ (94)
Verbesserung $>500 \%(94)$ 
schlechteste der Landkreis Vorpommern-Rügen. Auffällig sind die Landkreise in Sachsen-Anhalt und Mecklenburg-Vorpommern, die, entgegen dem bundesweiten Trend, eine Verschlechterung der Effizienz um mehr als $50 \%$ aufweisen.

Abbildung 3 präsentiert die Veränderung von Periode P3 zu P2. Der Median von $-52 \%$ signalisiert einen bundesweiten Trend der Verschlechterung der Effizienz. Lediglich die auch in Abbildung 2 auffälligen Bundesländer Sachsen-Anhalt und Mecklenburg-Vorpommern zeigen starke Verbesserungen. Hier erfolgte offensichtlich der Ausbauboom, der vielerorts zwischen 2005 und 2010 stattgefunden hat, erst in der nachfolgenden Periode. Dies lässt sich insbesondere durch die signifikant verbesserte Effizienz im Ausbau von Photovoltaik und Biogas erklären. ${ }^{14}$ Die stärkste Verbesserung verzeichnet in dieser Periode der Landkreis Rostock.

\subsubsection{Effizienzen im Ausbau von Windenergie}

Abbildung 4 stellt die Ausbaueffizienzen der Landkreise bezüglich der Windenergie dar. Hier ist ein deutliches Nord-Süd-Gefälle feststellbar. Zudem ist die große Streuung der Effizienzwerte auffällig (Spannweite = 17.13): Der Wert der höchsten beobachteten Effizienz liegt bei 1,14 (Landkreis Alzey-Worms), die größte Ineffizienz bei 17,13 für den Landkreis Rhön-Grabfeld. ${ }^{15}$ Der Median beträgt 6,85. Lediglich je ein Landkreis in Niedersachsen und Schleswig-Holstein belegen einen Platz unter den Top 10, obwohl diese beiden Bundesländer als die „Windmüller" Deutschlands gelten. ${ }^{16}$

Ebenfalls im Gegensatz zu der vorhergehenden Betrachtung der erneuerbaren Energieträger insgesamt kam es in der Periode 2005-2009 zu einem vermehrten Rückgang der Effizienz in nahezu ganz Deutschland: 210 Landkreise waren in der Periode weniger effizient als in der Vorperiode (Median $=-91,82 \%$ ). Besonders auffällig ist der Osten Deutschlands mit einer überproportionalen Verschlechterung von über $50 \%{ }^{17}$ Das bedeutet, dass der Abstand zwischen den führenden Regionen und allen anderen deutlich größer geworden ist. Die stärkste Verbesserung verzeichnete der Landkreis Lüchow-Dannenberg, die stärkste Verschlechterung der Landkreis Steinfurt.

14 Abbildungen B, C, D und E im online supplement.

15 Tabelle $C$ im online supplement.

$16 \mathrm{https} / / / \mathrm{www}$. wind-energie.de/themen/statistiken (10.04.2019).

17 Abbildung $\mathrm{F}$ im online supplement.
In der nachfolgenden Periode (P3) ist das Gegenteil zu beobachten, es kam wieder zu einer Verbesserung der Effizienz ${ }^{18}$ das heißt zu einem Aufholprozess. Auch hier stechen die östlichen Bundesländer hervor, dieses Mal jedoch durch eine starke Verbesserung der Effizienz. Ähnliches kann für südliche Landkreise Ostdeutschlands festgestellt werden. Der Median liegt in dieser Periode bei 17,70. Die stärkste Verbesserung erfuhr der Landkreis Schleswig-Flensburg, die größte Verschlechterung Altenkirchen (Westerwald). Auffällig ist jedoch, dass alle der letzten zehn Rangplätze sich in dieser Periode entgegen dem Trend verschlechtert haben. Generell lässt sich bei diesem Energieträger feststellen, dass die Regionen der hinteren Rangplätze nur geringe Schwankungen in ihrer Effizienz aufweisen, woraus zu schließen ist, dass es in diesen Landkreisen keinen merklichen Ausbau der Windenergie gegeben hat, sondern nur vergleichsweise inkrementell ausgebaut wurde.

\subsubsection{Regionale Ausbaueffizienz der Photovoltaik}

Abbildung 5 demonstriert die Ausbaueffizienzen der Landkreise in Bezug auf Photovoltaik bis zum Jahr 2014. Hier fallen die hohen Ineffizienzen im Osten Deutschlands auf, wo fast alle 43 Landkreise mit einem (In-) Effizienzwert größer 6 lokalisiert sind. Nur vereinzelt finden sich auch in Niedersachsen und Schleswig-Holstein Landkreise mit ähnlich hohen Werten. Die Streuung der Effizienzwerte ist geringer als beim Ausbau von Windenergie (Spannweite 10,19). Dies zeigt, dass die Landkreise beim Ausbau von Photovoltaik recht ähnlich effizient sind. Die größte Effizienz besitzt der Landkreis Steinfurt $(1,08)$, die geringste Mansfeld-Südharz $(11,28)$. Sieben der Top-10-Regionen liegen in Bayern. ${ }^{19}$

In der Periode 2005-2009 kam es nur in neun Landkreisen zu einer Verschlechterung der Effizienz verglichen mit der Vorperiode (P1) (Median $=26,97 \%$ ), was insbesondere für Landkreise in Mecklenburg-Vorpommern gilt. ${ }^{20} \mathrm{Am}$ stärksten verbessert hat sich das Emsland, am meisten verschlechtert der Altmarkkreis Salzwedel.

Der nationale Ausbauboom mit einem einhergehenden Angleichungsprozess hat sich jedoch in großen Teilen Deutschlands nicht fortgesetzt, was sich in der negativen Veränderung der Effizienz in der nachfolgenden Periode (P3) zeigt (Median $=-98 \%)$. Bemerkenswert sind dabei

18 Abbildung $\mathrm{G}$ im online supplement

19 Tabelle $D$ im online supplement.

20 Abbildung B im online supplement. 


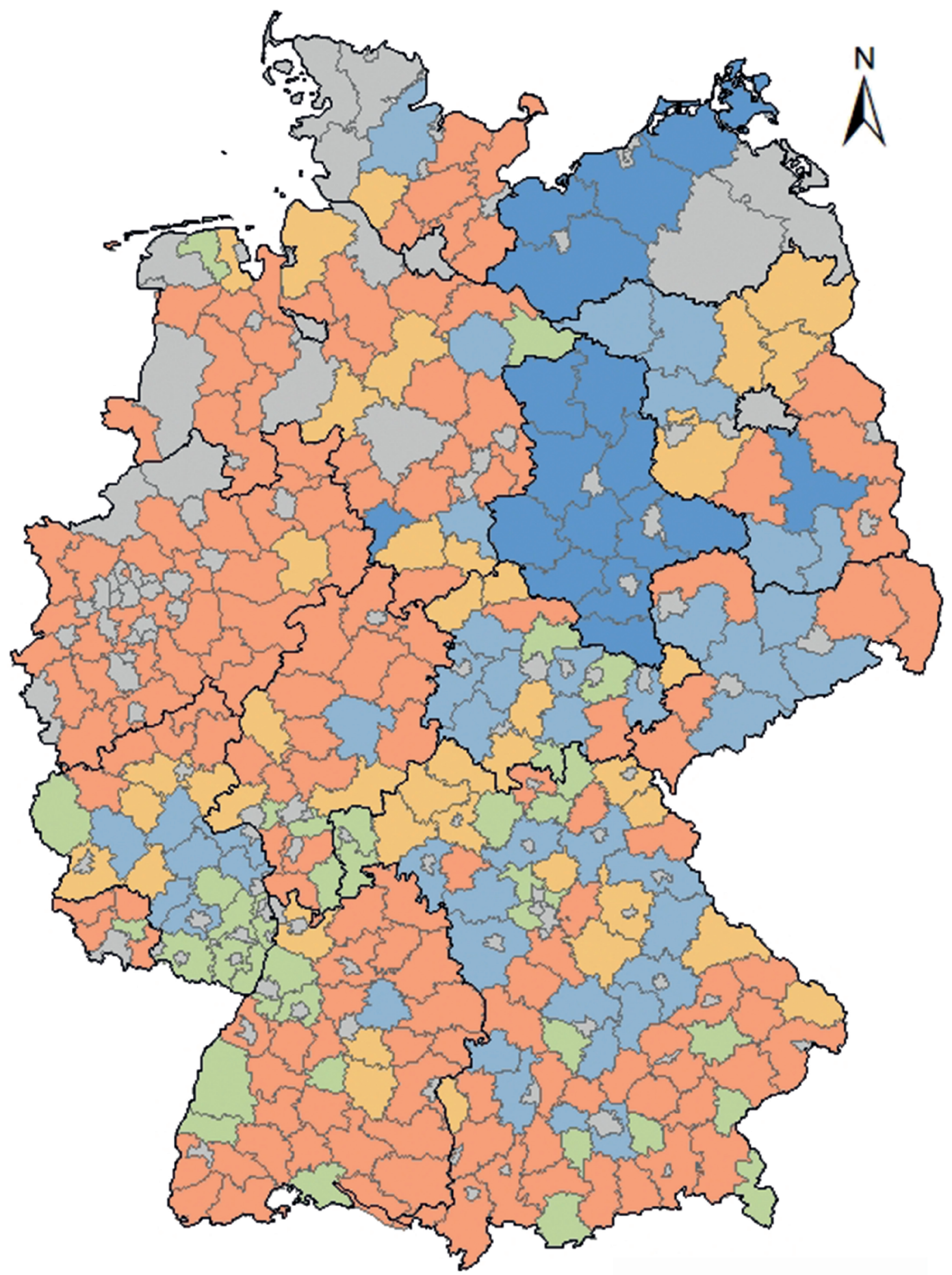

nicht betrachtet (121)

Verschlechterung > $50 \%$ (144)

Verschlechterung < = $50 \%(40)$

keine Veränderung (36)

Verbesserung <= $500 \%$ (44)

Verbesserung $>500 \%(17)$

$\begin{array}{lll}0 & 30 \quad 60 \quad 120 \\ \end{array}$ 


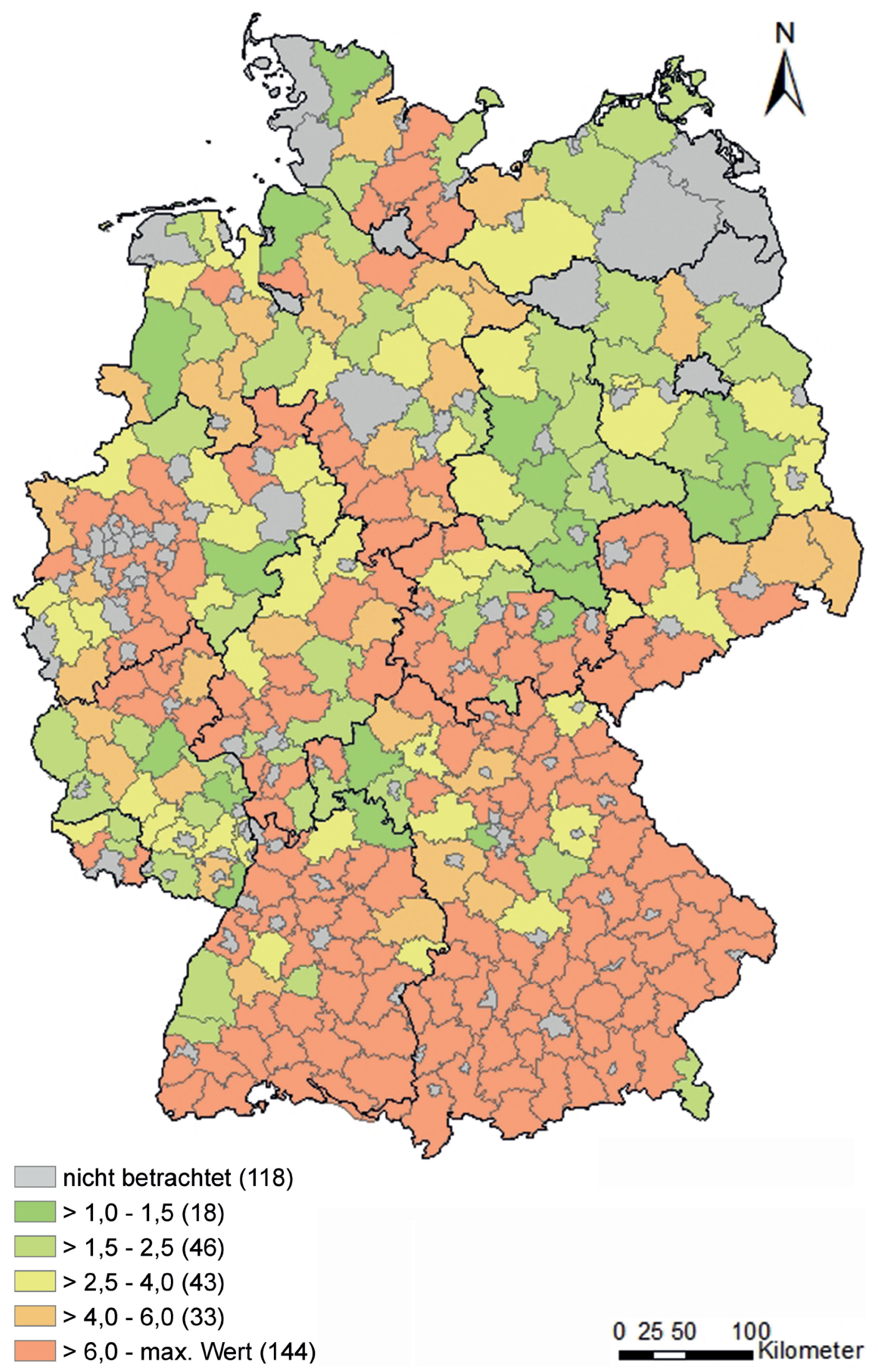

Abbildung 4: Robuste Effizienzwerte der Landkreise für den Ausbau von Windenergie 


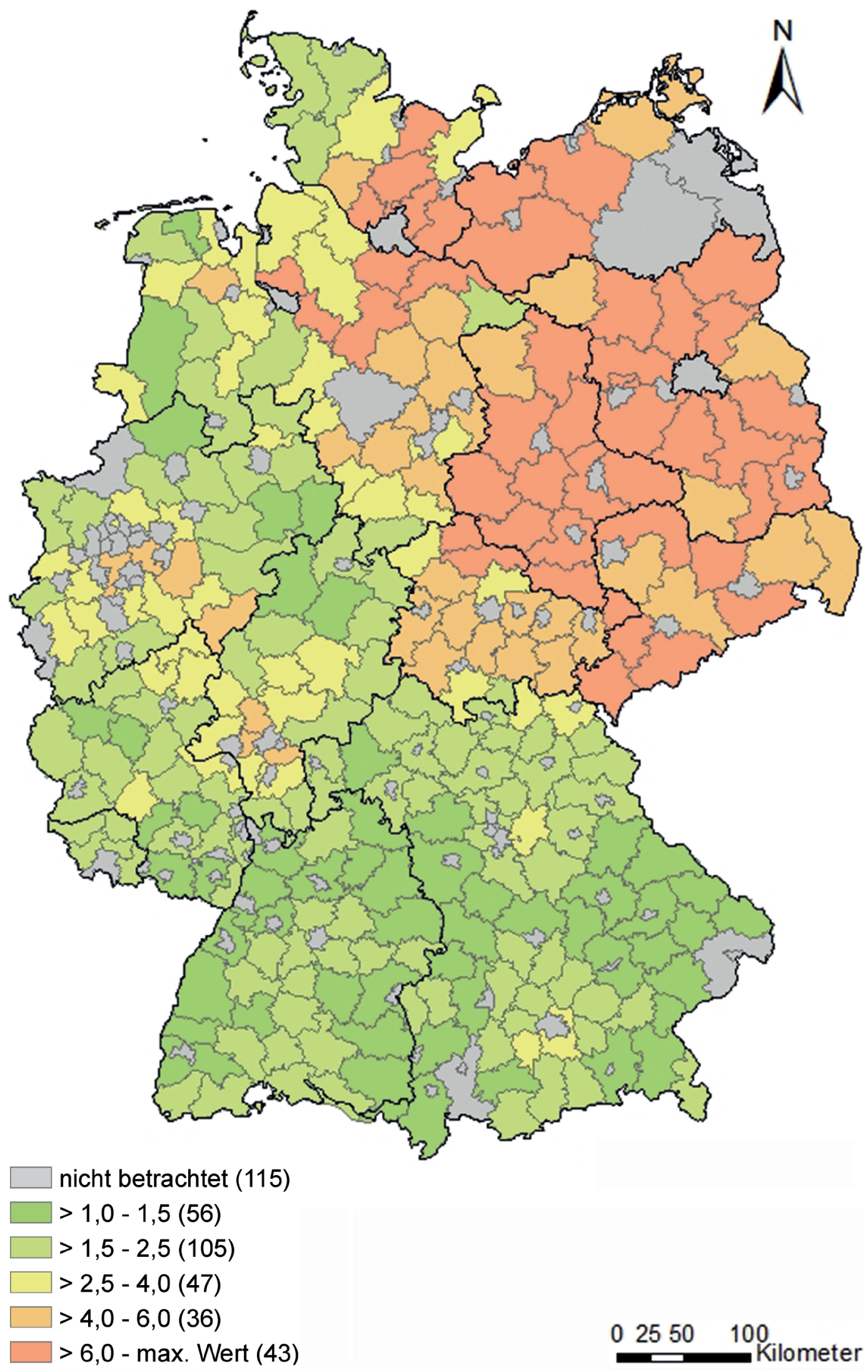

Abbildung 5: Robuste Effizienzwerte der Landkreise für den Ausbau von Photovoltaik 
die Bundesländer Sachsen-Anhalt und MecklenburgVorpommern, in denen es offensichtlich wieder zu einer starken Verbesserung der Effizienz kam. ${ }^{21}$ Auch fällt auf, dass sich, entgegen dem negativen Trend in Deutschland, sechs der zehn schlechtesten Landkreise beim Ausbau von Photovoltaik verbessert haben, was auf einen leichten Aufholprozess in dieser Gruppe der Regionen während dieser Periode hindeutet. Der Landkreis Steinfurt hat sich am meisten verschlechtert, der Landkreis VorpommernRügen hingegen am meisten verbessert.

\subsubsection{Regionale Ausbaueffizienzen von Biogas}

Unter den drei betrachteten Energieträgern weist Biogas die bundesweit besten Werte bei den regionalen Ausbaueffizienzen auf (Median=2,21). Die recht große Streuung ist durch die hohe Ineffizienz des letztplatzierten Kreises Rhein-Lahn (1.576) zu erklären. Zum Vergleich: An vorletzter Stelle steht der Oberbergische Kreis mit einem Effizienzwert von $41 .{ }^{22}$ Nur vereinzelt lassen sich Werte größer 6 in Mittel- oder Ostdeutschland beobachten (vgl. Abbildung 6). Die höchste Effizienz besitzt der Heidekreis $(1,08)$. Die ersten zehn Plätze belegen Landkreise aus Niedersachsen, Baden-Württemberg und Bayern.

Ähnlich wie beim Ausbau von Photovoltaik verbesserte sich zwischen 2005 und 2009 in fast allen Landkreisen die Effizienz (Median= 3.89). Lediglich 17 Landkreise haben sich in dieser Periode verschlechtert. ${ }^{23}$ Die Landkreise in Mecklenburg-Vorpommern fallen auch in dieser Periode wieder durch eine Verschlechterung der Effizienz auf. Die stärkste Verbesserung zeigen die Landkreise Cloppenburg, Rotenburg (Wümme) und Emsland, die stärkste Verschlechterung der Rhein-Lahn-Kreis.

In der darauffolgenden Periode (P3) verringerte sich die Effizienz (Median= -98). Ähnlich wie bei der Photovoltaik verbesserten sich die Landkreise in den Bundesländern Sachsen-Anhalt und Mecklenburg-Vorpommern entgegen dem negativen Trend in der späteren Periode, was wieder für einen Aufholprozess in diesen Bundesländern spricht. ${ }^{24} \mathrm{Am}$ meisten verbessert hat sich der Landkreis Ludwigslust-Parchim, während sich der Landkreis Emsland am stärksten verschlechtert hat.

21 Abbildung $C$ im online supplement.

22 Tabelle E im online supplement.

23 Abbildung $D$ im online supplement.

24 Abbildung $\mathrm{E}$ im online supplement.

\subsection{Experteninterviews}

Zur Validierung der quantitativen Ergebnisse der Effizienzbestimmung und zur Ermittlung möglicher Ursachen der regionalen Effizienzunterschiede wurden insgesamt acht Experten in Niedersachsen befragt. Da sich gesetzliche und politische Rahmenbedingungen zwischen den Bundesländern unterscheiden können, aber innerhalb desselben Bundeslandes relativ homogen sind, beschränkt sich die vertiefende qualitative Empirie auf ein Bundesland. Aus forschungspraktischen und ressourcenbezogenen Gründen fiel die Entscheidung auf das Flächenland Niedersachsen. Genauer betrachtet wurde der Landkreis Hameln-Pyrmont sowie der Heidekreis - beide fallen vor allem durch hochrangige Platzierungen beim Ausbau von Biogas auf - sowie die Landkreise Holzminden, Harburg und Vechta, die eher im letzten Drittel der 281 betrachteten Landkreise platziert sind. Die Experten waren zum Zeitpunkt der Erhebungen entweder für den jeweiligen Landkreis oder in einer Klimaschutzleitstelle tätig. Zusätzlich wurde ein Vorstandsmitglied aus einem Bürgersolarkraftwerk sowie Aufsichtsratsmitglied einer Energiegenossenschaft als Experte für den Photovoltaik-Ausbau befragt, da dieser Energieträger in allen fünf Landkreisen eher schlecht bewertet wurde. Ausgangspunkt der qualitativen Interviews sind Einzelfallbeschreibungen, die keinen Anspruch auf Repräsentativität haben. Sie können daher auch nur für eine generelle Beurteilung der Methodik bzw. eine Typisierung der Fälle verwendet werden.

Die verwendete Methode der Effizienzmessung (DEA) war allen Experten unbekannt, weshalb sie zu Beginn in einer kurzen Präsentation erläutert wurde. Alle Experten erkannten die Wichtigkeit der Berücksichtigung des Ausbaupotenzials bei der Bewertung des aktuellen Ausbaustandes an. Das Fehlen eines entsprechenden regionalen Vergleichs wurde ebenfalls bemängelt, was die Signifikanz der von uns identifizierten Forschungslücke unterstreicht.

Den Experten fiel auf, dass mit dem Input-Faktor "Landwirtschaftliche Fläche" die Windeignungsfläche überschätzt wird. Zwar stünden die meisten Anlagen auf solchen Flächen, dennoch müssten Abstände zu Gebäuden eingehalten werden, die zwischen den Landkreisen variieren. Landkreise mit einer starken Zersiedelung auf recht engem Raum sind daher potenziell schlechter gestellt als Landkreise mit großen Landwirtschaftsflächen und wenig Bebauung. Auch artenschutzrechtliche Bedenken an Flussniederungen verringern das Potenzial, wenn für diese Flächen kein Schutzstatus besteht. In einem Interview wurde angesprochen, dass 


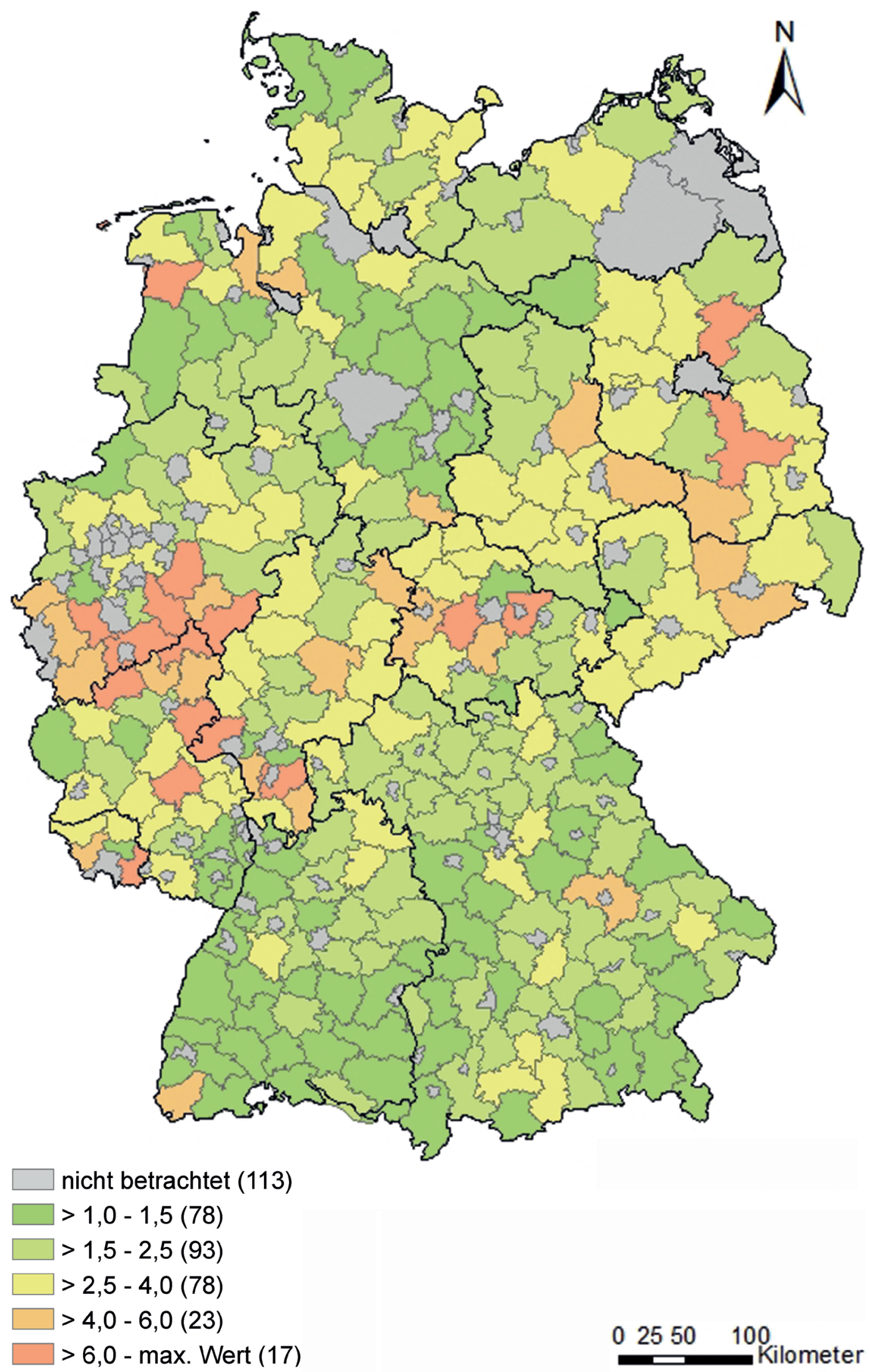


sich der Anbau von Bioenergiepflanzen aufgrund der guten Bodenqualität im Landkreis Vechta nicht lohne. Allerdings widerspricht diese Aussage der Verteilung der Bodengüte und den Biogaseffizienzwerten: Auch Landkreise mit extrem hoher Bodengüte, wie beispielsweise Hildesheim in Niedersachsen ${ }^{25}$, weisen in der quantitativen Analyse sehr gute Effizienzwerte auf. In einem weiteren Interview wurde ein allgemeiner Datenfehler im Windenergiebereich aufgedeckt, der anschließend durch Neuberechnungen der Effizienzwerte eliminiert wurde. Nur in einem Interview gab es eine Diskrepanz zwischen den Expertenkenntnissen zur Installation von Windenergieanlagen und den übermittelten Daten von EnergyMap. An dieser Stelle wurde auch deutlich, dass es selbst für die Experten im jeweiligen Landkreis oftmals schwierig ist, genaue Daten über die Ausbaustände zu erhalten. Von diesen Anmerkungen, die in zukünftiger Forschung Berücksichtigung finden sollten, abgesehen, erschien die hier getroffene Auswahl an Input-Faktoren den Experten insgesamt plausibel.

Die ermittelten empirischen Ausbaueffizienzergebnisse stimmten grundsätzlich mit den Kenntnissen der Experten überein. So wurde beispielsweise der allgemeine Rückgang der Ausbauintensität seit 2009 bestätigt. Gründe hierfür waren die EEG-Novellierungen und damit verringerte Fördermöglichkeiten sowie schärfere Anforderungen, aber auch teils veraltete Regionale Raumordnungsprogramme (RROP), deren Vorranggebiete bereits bebaut waren. Wegen des Mangels an ausgewiesenen Flächen, vor allem für Windenergie, führten die Unsicherheiten zu einem Investitionsstopp. Dies könnte eine Erklärung für den starken Rückgang der Ausbaueffizienz zwischen 2005 und 2009 sein, wobei sich allerdings die Frage stellt, warum dies nicht in allen Regionen in gleichem Maße zu beobachten war. Dies sollte in zukünftigen Arbeiten angegangen werden. Die Erschöpfung des Potenzials durch einen rasanten Ausbau in den vorangegangenen Jahren wurde als Erklärung für den Rückgang der Ausbaueffizienz angeführt.

Die genauer analysierten Landkreise verfolgen keine bewusste Spezialisierung auf einen bestimmten Energieträger. Vielmehr kam es zum Ausbau von bestimmten Energieerzeugungsarten in Abhängigkeit von Förderprogrammen.

Die sehr guten Ergebnisse des Heidekreises sind überwiegend auf findige Landwirte zurückzuführen, die sich in verschiedenen Arbeitsgruppen organisiert haben.

25 Vgl. http://www.nls.niedersachsen.de/Tabellen/Landwirtschaft/ nutzungen/artikel_1_2001.htm\#S (10.04.2019).
Im Landkreis Hameln-Pyrmont ist die hohe Effizienz im Biogasausbau mit der Teilnahme am Bundeswettbewerb Bioenergie-Regionen zu erklären. Die geringen Effizienzwerte im Bereich Photovoltaik werden mit dem Mangel an größeren Freiflächenanlagen begründet.

Die qualitativen Ergebnisse bestätigen somit die Ergebnisse und die Adäquanz des empirischen Ansatzes. Alle Experten erkannten den Mehrwert der empirischen Arbeit an (Berücksichtigung der Ausbaupotenziale) und schätzten den zusätzlichen Erkenntnisgewinn. Gerade die Identifikation von Best-practice-Regionen und die Informationen zur Größe der relativen Unterschiede wurden dabei herausgestellt. Die in den Interviews aufgedeckten Mängel wurden, soweit es die Datenlage erlaubte, behoben und somit die Verlässlichkeit der Analyse weiter erhöht. Insgesamt stellt die in einigen Teilen unbefriedigende Datenlage (Zersiedelung) den wichtigsten Kritikpunkt dar. Dies kann eventuell in zukünftigen Arbeiten behoben werden.

\section{Diskussion, Limitationen, politische Implikationen}

Die in Kapitel 4 dargestellten Ergebnisse zeigen, dass regionale Unterschiede in der Effizienz des Ausbaus erneuerbarer Energien existieren. Diese regionalen Unterschiede sind nicht allein auf variierende geographische und sozioökonomische Eigenschaften zurückzuführen, denn innen wurde in der quantitativen Analyse explizit Rechnung getragen. Die identifizierten räumlichen Differenzen in der Ausbaueffizienz sind vielmehr zu erheblichen Teilen mit Unterschieden in der sozialen Akzeptanz und dem politischen Willen zu erklären. Diesbezüglich bestätigt der Beitrag für die kleinräumige Ebene deutscher Landkreise die Erkenntnisse von Hoogwijk (2004) und Diekmann/Schill/Püttner et al. (2017).

Obwohl das primäre Ziel des Beitrages die Identifizierung von Unterschieden in der Ausbaueffizienz auf kleinräumiger Ebene ist, sind auch Differenzen auf der Bundesländerebene offensichtlich. So fällt beispielsweise die Ineffizienz östlicher Regionen beim Ausbau von Photovoltaik auf, wobei nicht abschließend geklärt werden konnte, ob diese historisch-politischen (z. B. Unterschiede in der regionalen Förderung durch das 10.000-Häuser-Programm in Bayern ${ }^{26}$ ) oder soziogeographischen (Unterschiede beim Ausmaß der Zersie-

26 https://www.energieatlas.bayern.de/buerger/10000_haeuser programm.html (10.04.2019). 
delung und des verfügbaren Einkommens) Ursprungs sind. Zudem besteht bei Photovoltaik eine Flächenkonkurrenz zu Solarthermieanlagen. Leider gibt es für sie keine Daten auf der Kreisebene. Eine Auswertung auf der Ebene von Bundesländern deutet allerdings eher auf eine positive Beziehung zwischen den beiden Systemen hin. ${ }^{27}$ Nichtsdestotrotz können wir nicht abschließend evaluieren, welchen Einfluss die Flächenkonkurrenz auf unsere Ergebnisse hat.

Wir nehmen vereinfachend an, dass die auf Bundesund EU-Ebene gesetzten Rahmenbedingungen für den Ausbau erneuerbarer Energien für alle Regionen identisch sind. Inwieweit diese Annahme gerechtfertigt ist, bleibt den Ergebnissen zukünftiger Forschung überlassen.

Ähnlich den Ergebnissen von Diekmann, Schill, Püttner et al. (2017) erweisen sich auch im vorliegenden Beitrag die Landkreise in Bayern und Baden-Württemberg insgesamt als sehr effizient, was vor allem mit den hohen Effizienzwerten beim Ausbau von Photovoltaikund Biogasanlagen begründet werden kann. Die Ineffizienzen der Landkreise dieser Bundesländer beim Ausbau von Windenergie werden hierdurch kompensiert. Ostdeutsche Landkreise erreichen hingegen in der Gesamtbetrachtung hohe Werte beim Windenergieausbau und mittlere Effizienzwerte beim Biogasanlagenausbau. Überraschend ist, dass Niedersachsen und SchleswigHolstein, die als ,landwirtschaftlicher Motor und Windmüller Deutschlands' gelten, in der Gesamtbetrachtung nicht übermäßig positiv auffallen, was zumindest partiell mit geringen Effizienzwerten beim Photovoltaik-Ausbau zu erklären ist. Das sehr große Flächenpotenzial dieser Länder ist also noch vergleichsweise wenig ausgeschöpft, zumindest im relativen Vergleich mit Landkreisen anderer Bundesländer. Auffällig ist zudem Sachsen, dessen Landkreise besonders geringe Effizienzwerte bei allen Energieträgern aufweisen.

Die differenzierte Betrachtung einzelner Energieträger hat deutlich gemacht, dass innerhalb eines Landkreises meist nur ein Energieträger stark ausgebaut wurde, was auf Spezialisierungstendenzen hindeutet. Dem widersprechen die Ergebnisse der Experteninterviews für ausgewählte niedersächsische Landkreise, in denen keine absichtliche Spezialisierung bestätigt wurde. Vielmehr scheinen diese über die Zeit aus vorhandenen Strukturen und nichtintendierten Pfadabhängigkeiten entstanden zu sein.

27 Vgl. https://www.solarwirtschaft.de/fileadmin/user_upload/ DEPV_Grafik_Anteil_3Heizsysteme_D.pdf (10.04.2019).
Die vorliegenden Ergebnisse müssen im Lichte der empirischen Limitationen des Beitrages interpretiert werden. Über die in Kapitel 3.2.2 genannte Kritik an der verwendeten Effizienzanalyse (DEA) hinaus sind die Effizienzwerte in Teilen durch die vorangegangene Ausreißeranalyse geprägt. Die Entscheidung zum Ausschluss oder zur Berücksichtigung bestimmter Beobachtungen ist letztlich immer eine subjektive Entscheidung, die zwar nach bestem Wissen und Gewissen durchgeführt wurde, aber natürlich nicht an ein objektives Kriterium heranreicht. Hier bedarf es zukünftig einer noch verlässlicheren Datenbasis sowie weiterer methodologischer Fortschritte, die den Grad der Subjektivität verringern. Das betrifft insbesondere die mögliche Überschätzung einiger Input-Faktoren wie "Landwirtschaftliche Fläche“ oder "Gebäude- und Freifläche“, die von den befragten Experten genannt wurde.

Gerade die einzuhaltenden Abstände zu Wohnund Naturschutzgebieten können die potenzielle Fläche erheblich reduzieren. Allerdings sind dies im Regelfall lokalpolitische Festlegungen. Beispielsweise gilt seit 2014 in Bayern die 10-H-Regelung, die festlegt, dass das Zehnfache der Anlagenhöhe als Mindestabstand zu Wohnbebauung eingehalten werden muss. Dadurch reduziert sich die mögliche Fläche für Windräder auf 0,01 Prozent der Landesfläche. Im Sinne dieser Studie stellt dies jedoch keine grundsätzliche Einschränkung des (Windkraftanlagen-)Ausbaupotenzials dar. Es ist vielmehr eine bewusste politische Entscheidung, das vorhandene Potenzial nicht auszuschöpfen (das heißt, eine niedrige Ausbaueffizienz zu haben) und sich dafür potenziell in anderen Bereichen (z. B. Wohnqualität und Umweltschutz) zu verbessern. Vor diesem Hintergrund ist es wichtig zu berücksichtigen, dass ein Maximieren der Ausbaueffizienz erneuerbarer Energien nur eines von vielen (in Teilen konkurrierenden) politischen Zielen ist.

Auch der Faktor "Gebäude- und Freifläche“ überschätzt die potenziellen Flächen, da bei Photovoltaik Neigung und Ausrichtung der Dachflächen ebenfalls eine Rolle spielen. Die Berücksichtigung der „durchschnittlichen Hangneigung" bei den Berechnungen trägt zwar der unterschiedlichen Topographie der Regionen Rechnung, sie weist aber gerade in Bezug auf Photovoltaik-Anlagen Unschärfen auf. Zwar können bei steiler Hangneigung keine Photovoltaik-Anlagen auf Freiflächen installiert werden, dieses Argument verliert jedoch an Gewicht, wenn die Hangflächen mit Gebäuden bebaut sind. Ebenso unbeachtet blieb das Potenzial von Konversionsflächen und von Freiflächen entlang von Autobahnen oder Eisenbahntrassen. Ohne eine verbesserte Datenbasis können diese Aspekte jedoch 
weder berücksichtigt noch bezüglich ihrer empirischen Relevanz überprüft werden.

Für die Effizienzberechnung wurde versucht, einen möglichst langen Zeitraum zu berücksichtigen. Prämisse war, dass die regionalen Potenziale über die Zeit konstant bleiben. Es ist aber denkbar, dass die Input-Faktoren sich partiell verändern, wodurch Landkreise möglicherweise nicht adäquat bewertet wurden. Beispiel hierfür ist der in Kapitel 2.1 erwähnte Umbruch von Grünland im Rahmen der Ausdehnung der Biogasproduktion. Auch der Vieh- und Bevölkerungsstand wird sich über die Zeit verändert haben. Wie auch von den Experten betont, ist der Ausbau aber primär von rechtlichen Rahmen- und den schwankenden Förderbedingungen abhängig, was im Beitrag durch das gewählte methodische Vorgehen explizit berücksichtigt wurde. Folglich sollte der Einfluss der genannten Schwankungen der Input-Faktoren auf das Ergebnis nur gering sein.

Angesichts der Tatsache, dass bisher die Potenziale subnationaler Regionen zum Ausbau von erneuerbaren Energien bei der Evaluierung des Ausbaustandes überhaupt nicht berücksichtigt wurden, sollten die genannten Datenprobleme allerdings auch nicht überbewertet werden.

Ein weiterer innovativer Aspekt des vorliegenden Beitrags ist die Validierung der quantitativen empirischen Ergebnisse durch eine nachgelagerte qualitative Analyse. So haben die Interviews nicht nur den Nutzen der empirischen Arbeit unterstrichen, sondern auch bestätigt, dass es offenbar keine einzelne Ursache für die Effizienzunterschiede beim Ausbau erneuerbarer Energien gibt. Es ist vielmehr das Zusammenspiel vieler Faktoren, das die Situation eines Landkreises erklärt. Mit den Worten von Linder (2013: 84): „Räumliche Muster sind häufig das Ergebnis sozialer Prozesse, die nicht allein durch die Eigenschaften einzelner Individuen erklärt werden können, sondern vielmehr durch die Interaktionen von Individuen im Raum." Die meisten befragten Experten zeigten großes Interesse an den Ergebnissen der Analyse und bestätigten, dass diese als „Stärken-SchwächenAnalyse" in der politischen Diskussion um die Ausrichtung des Landkreises im Ausbau von erneuerbaren Energien genutzt werden können, da von ihnen eine große Außenwirkung ausgehe. In diesem Sinne stellt der Beitrag einen neuen Ansatz dar, der bestehende Methoden ergänzen, aber nicht ersetzen kann.

Die empirischen Befunde des Beitrages erlauben einige politische Schlussfolgerungen. Erstens existieren ganz offensichtlich regionale Unterschiede in der Ausschöpfung der Potenziale regenerativer Energien. Diese Unterschiede lassen sich aus räumlicher Perspektive zwi- schen Landkreisen innerhalb desselben Bundeslandes und zwischen (Landkreisen in) Bundesländern beobachten. Da im vorliegenden Beitrag wichtige Determinanten dieses Ausschöpfungsgrades kontrolliert wurden, sind die Ergebnisse interregional vergleichbar. Ergo können sich Landkreise mit (bislang) vergleichsweise geringer Ausschöpfung ihrer Potenziale an anderen Landkreisen orientieren, die ähnliche Voraussetzungen, aber bessere Effizienzen aufweisen. Dies sollte von der Lokalpolitik dieser Landkreise, aber auch von der zugehörigen Landespolitik als Handlungsbedarf interpretiert werden. Zweitens zeigt der Beitrag zusätzlich zu den genannten räumlichen Disparitäten der Ausbaueffizienz auch landkreisbezogene Unterschiede bei der Art der drei betrachteten regenerativen Energieträger. Die Schlussfolgerungen bezüglich des politischen Handlungsbedarfs sind dieselben wie beim ersten Argument: Lokal- und Landespolitik sollten sich solche Landkreise und Bundesländer als Vorbild nehmen, die bei ähnlichen (oder gar ungünstigeren) Rahmenbedingungen bei einzelnen regenerativen Energieträgern deutlich höhere Effizienzen erzielen. Beide Arten von Lerneffekten können in der Summe aller deutschen Landkreise einen spürbaren Beitrag zum Gelingen der Energiewende leisten.

\section{Literatur}

Arbach, S.; Gerlach, A.-K.; Kühn, P.; Pfaffel, S. (2013): Entwicklung der Windenergie in Deutschland. Eine Beschreibung von aktuellen und zukünftigen Trends und Charakteristika der Einspeisung von Windenergieanlagen. Kassel.

Backhaus, K.; Wilken, R. (2003): Effizienzmessung im Marketing mit Data-Envelopment-Analysis: Eine methodische Bestandsaufnahme. Münster. = Arbeitspapiere des betriebswirtschaftlichen Instituts für Anlagen und Systemtechnologien 34 .

Banker, R. D.; Charnes, A.; Cooper, W. W. (1984): Some models for estimating technical and scale inefficiencies in data envelopment analysis. In: Management Science 30, 9, 10311142. doi: $10.1287 / \mathrm{mnsc} .30 .9 .1078$

BMWI - Bundesministerium für Wirtschaft und Energie (2018): Erneuerbare Energien in Zahlen. Nationale und internationale Entwicklung im Jahr 2017. Berlin.

Broekel, T.; Alfken, C. (2015): Gone with the wind? The impact of wind turbines on tourism demand. In: Energy Policy 86, 506519. doi: 10.1016/j.enpol.2015.08.005

Bundesverband Windenergie (2013): Energie in Bürgerhand. Energie aus der Region für die Region. Berlin.

Cantner, U.; Krüger, J.; Hanusch, H. (2007): Produktivitäts- und Effizienzanalyse. Der nichtparametrische Ansatz. Berlin. doi: 10.1007/978-3-540-70794-3 
Cazals, C.; Florens, J.-P.; Simar, L. (2002): Nonparametric frontier estimation: a robust approach. In: Journal of Econometrics 106, 1, 1-25. doi: 10.1016/S0304-4076(01)00080-X

Daraio, C.; Simar, L. (2007): Advanced Robust and Nonparametric Methods in Efficiency Analysis: Methodology and Applications. New York. = Studies in Productivity and Efficiency 4.

Diekmann, J.; Schill, W.-P.; Püttner, A.; Kirrmann, S. (2017): Vergleich der Bundesländer: Analyse der Erfolgsfaktoren für den Ausbau der Erneuerbaren Energien 2017: Indikatoren und Ranking. Berlin. = DIW Politikberatung kompakt 125.

Dorfard, A. (2014): Kosteneffizienzen und Einsparungspotenziale durch Fusionen: Eine Anwendung auf die Kommunal- und Verwaltungsreform in Rheinland-Pfalz. Wiesbaden. doi: 10.1007/978-3-658-01501-5

European Commission (2010): Europe 2020. A strategy for smart, sustainable and inclusive growth. Brüssel.

Flick, U. (1991): Handbuch qualitative Sozialforschung: Grundlagen, Konzepte, Methoden und Anwendungen. München.

Franke, P. (2014): Tücken des Geländes. In: Photovoltaik 10, 44-48.

Glaser, B. G.; Strauss, A. L. (1998): Grounded theory: Strategien qualitativer Forschung. Bern.

Günther, M. (2015): Energieeffizienz durch Erneuerbare Energien: Möglichkeiten, Potenziale, Systeme. Wiesbaden. doi: 10.1007/978-3-658-06753-3

Hammerschmidt, M.; Wilken, R.; Staat, M. (2009): Methoden zur Lösung grundlegender Probleme der Datenqualität in DEAbasierten Effizienzanalysen. In: Die Betriebswirtschaft 69, 2, 289-309.

Hoogwijk, M. A. (2004): On the global and regional potential of renewable energy sources. Utrecht.

Jami, A.A.N.; Walsh, P.R. (2014): The role of public participation in identifying stakeholder synergies in wind power project development: The case study of Ontario, Canada. In: Renewable Energy 68, 194-202. doi: 10.1016/j.renene.2014.02.004

Keppler, D. (2007): Regionale Einflussfaktoren auf den Ausbau erneuerbarer Energien. Ergebnisse einer Fallstudie in der Brandenburger Energieregion Lausitz. In: Gaia 16, 4, 289-296.

Kerpen, P. (2016): Praxisorientierte Data Envelopment Analysis. Wiesbaden. doi: 10.1007/978-3-658-15460-8

Kontogianni, A.; Tourkolias, C.; Skourtos, M.; Damigos, D. (2014): Planning globally, protesting locally: Patterns in community perceptions towards the installation of wind farms. In: Renewable Energy 66, 170-177. doi: 10.1016/j. renene.2013.11.074

Linder, S. (2013): Räumliche Diffusion von Photovoltaik-Anlagen in Baden-Württemberg. Würzburg. = Würzburger Geographische Arbeiten 109

Lödl, M.; Kerber, G.; Witzmann, R.; Hoffmann, C.; Metzger, M. (2010): Abschätzung des Photovoltaik-Potentials auf Dachflächen in Deutschland. München. https://mediatum. ub.tum.de/doc/969497/969497.pdf (08.04.2019).

Lütkehus, I.; Salecker, H.; Adlunger, K. (2013): Potenzial der Windenergie an Land. Studie zur Ermittlung des bundesweiten Flächen- und Leistungspotenzials der Windenergienutzung an Land. Dessau-Roßlau.

Miles, M. B.; Huberman, A. M.; Saldaña, J. (2014): Qualitative data analysis: A methods sourcebook. Los Angeles.
Padberg, T.; Werner, T. (2005): Analyse der Mehrjahresentwicklung der Effizienz von Sparkassen unter Einsatz der Data Envelopment Analysis und des Malmquist-Index. In: Schmalenbachs Zeitschrift für betriebswirtschaftliche Forschung 57, 3, 214-236. doi: 10.1007/BF03372764

Quaschning, V. (2016): Sektorkopplung durch die Energiewende. Anforderungen an den Ausbau erneuerbarer Energien zum Erreichen der Pariser Klimaschutzziele unter Berücksichtigung der Sektorkopplung. Berlin.

Rauner, S.; Eichhorn, M.; Thrän, D. (2016): The spatial dimension of the power system: Investigating hot spots of Smart Renewable Power Provision. In: Applied Energy 184, 1038-1050. doi: 10.1016/j.apenergy.2016.07.031

Rohrig, K. (2014): Wind Energy Report Germany 2014. Kassel.

Scheel, H. (2000): Effizienzmaße der Data Envelopment Analysis. Wiesbaden. doi: 10.1007/978-3-663-08017-6

Schütte, R. (2012): EEG stellt Kulturlandschaft auf den Kopf. https:// docplayer.org/63955342-Eeg-stellt-kulturlandschaft-auf-denkopf.html (07.05.2019).

Simar, L. (2007): How to improve the performances of DEA/FDH estimators in the presence of noise? In: Journal of Productivity Analysis 28, 3, 183-201. doi: 10.1007/s11123-007-0057-3

Simar, L.; Wilson, P. W. (1998): Sensitivity Analysis of Efficiency Scores. How to Bootstrap in Nonparametric Frontier Models. In: Management Science 44, 1, 49-61.

Weimann, J. (2012): Atomausstieg und Energiewende: Wie sinnvoll ist der deutsche Alleingang? In: Energiewirtschaftliche Tagesfragen 62, 12, 34-38.

Witt, J.; Thrän, D.; Rensberg, N.; Hennig, C.; Naumann, K.; Billig, E.; Sauter, P.; Daniel-Gromke, J.; Krautz, A.; Weiser, C.; Reinhold, G.; Graf, T. (2012): Monitoring zur Wirkung des Erneuerbare- Energien-Gesetz (EEG) auf die Entwicklung der Stromerzeugung aus Biomasse. Endbericht zur EEG-Periode 2009 bis 2011, Leipzig.

Wunderlich, C. (2012): Akzeptanz und Bürgerbeteiligung für Erneuerbare Energien. Er-kenntnisse aus Akzeptanz- und Partizipationsforschung. Berlin. $=$ Renews Spezial 60 . 